\title{
Comparison of two crossover procedures for describing thermodynamic behavior of normal alkanes from singular critical to regular classical regions
}

\author{
Jamali, Asma; Vinhal, Andre P. C. M.; Behnejad, Hassan; Yan, Wei; Kontogeorgis, Georgios M.
}

Published in:

Fluid Phase Equilibria

Link to article, DOI:

10.1016/j.fluid.2019.04.030

Publication date:

2019

Document Version

Peer reviewed version

Link back to DTU Orbit

Citation (APA):

Jamali, A., Vinhal, A. P. C. M., Behnejad, H., Yan, W., \& Kontogeorgis, G. M. (2019). Comparison of two crossover procedures for describing thermodynamic behavior of normal alkanes from singular critical to regular classical regions. Fluid Phase Equilibria, 495, 33-46. https://doi.org/10.1016/j.fluid.2019.04.030

\section{General rights}

Copyright and moral rights for the publications made accessible in the public portal are retained by the authors and/or other copyright owners and it is a condition of accessing publications that users recognise and abide by the legal requirements associated with these rights.

- Users may download and print one copy of any publication from the public portal for the purpose of private study or research.

- You may not further distribute the material or use it for any profit-making activity or commercial gain

- You may freely distribute the URL identifying the publication in the public portal 


\title{
Comparison of two crossover procedures for describing thermodynamic behavior of normal alkanes from singular critical to regular classical regions
}

\author{
Asma Jamali $^{\mathrm{a}, \mathrm{b}}$, Andre P.C.M.Vinhal ${ }^{\mathrm{b}}$, Hassan Behnejad ${ }^{\mathrm{a}^{*}}$, WeiYan ${ }^{\mathrm{c}}$, Georgios M.Kontogeorgis ${ }^{\mathrm{b}{ }^{*}}$ \\ ${ }^{a}$ Department of Physical Chemistry, School of Chemistry, University College of Science, \\ University of Tehran, Tehran 14155, Iran \\ ${ }^{b}$ Center for Energy Resources Engineering (CERE), Department of Chemical and Biochemical Engineering, \\ Technical University of Denmark, DK-2800 Kgs. Lyngby, Denmark \\ ${ }^{c}$ Center for Energy Resources Engineering (CERE), Department of Chemistry, Technical University of Denmark, \\ DK-2800 Kgs. Lyngby, Denmark
}

\begin{abstract}
In this work, two crossover procedures were applied to the Soave-Redlich-Kwong (SRK) equation of state $(E O S)$ in order to describe the thermodynamic behavior of hydrocarbons from far away up to close to the critical point. The first one is based on a renormalization group theory method, which uses a recursive procedure originally proposed by White and coworkers (Salvino and White, J. Chem. Phys. 96 (1992) 4559-4568). The second one incorporates the scaling laws close to the critical point into the cubic $E o S$, and was developed by Kiselev (Kiselev, Fluid Phase Equilibria, 147 (1998) 7 23). The classical and crossover $S R K E O S$ are applied to describe the phase behavior of pure n-alkanes (from methane to n-decane), and the comparison with experimental data indicates that the non-meanfield models are superior to the classical one for the representations of vapor-liquid coexistence data, isothermal pressure-density data and critical properties. Additionally, a thorough comparison of the two crossover approaches is done indicating the advantages and disadvantages of each approach.
\end{abstract}

Keywords: Crossover, Critical point, n-alkanes, Phase equilibrium 


\section{Introduction}

In the past decades, there has been a growing interest in clarifying the universal behavior of thermodynamic systems in the vicinity of the critical point. Close to the critical point, the divergence of some thermodynamic quantities become system independent, i.e., the critical exponents describing the divergence behavior are universal and their values depend only on the dimensionality of the problem. Besides, the exponent values are not related to the thermodynamic path, e.g., the critical exponent along the critical isochore corresponds to the critical exponent along the critical isotherm for a given property. This is due to the presence of long-range fluctuations in the order parameter $[1,2]$. The spatial extent of these critical fluctuations is theoretically determined by the correlation length and the divergence of correlation length can be observed experimentally through the critical opalescence phenomenon [3]. Consequently, the microscopic details of short-range intermolecular interactions become insignificant when the system is sufficiently close to the critical point [4].

The mathematical nature of the asymptotic and singular behavior of fluids near the critical point can be described by scaling laws given by the 3D-Ising model [1,5]. However, at conditions far away from the critical point, a transition from a singular to a classical mean field behavior is observed [6]. Mean-field theory models, like cubic equations of state $(E o S s)[7,8]$, fail to correctly represent the properties of fluids at the critical region because of the averaging process over a large number of molecules based on the statistical formalism that leads to ignoring the fluctuations inherent to the critical region [9]. However, from an engineering perspective, cubic $E o S$ are still widely applied in the simulation of industrial processes because of its simplicity and due to fast and reliable representations of the properties of pure components and mixtures. Therefore, in order to improve the reliability of the design and operation control of industrial processes, it is important to develop models that are capable of predicting the phase equilibrium of complex systems by bridging the gap between the anomalous behavior of systems close to the critical point and classical behavior sufficiently far away from the critical region $[7,10,11]$.

There are, in the literature, two main procedures to correct the classical models for representing the singular and asymptotic critical behavior of systems. They give a precise description of a fluid close to and far away from the critical point over a wide range of PVT data. The first successful attempt was employed by Chen et al. [12], and it was based on the renormalization group theory (RGT). Following this work, Sengers and coworkers [13] proposed the renormalized Landau 
expansion procedure. This procedure developed by Kiselev [14,15], in a simplified way contains a revised and extended equation for the Helmholtz free energy involving a crossover function. This function renormalizes the temperature and volume variables, incorporating the scaling law behavior of thermodynamic properties sufficiently close to the critical point. This procedure has been successfully applied to several classical EoS including cubic and SAFT (statistical associating fluid theory) EoS [7-9,15-18]. The second procedure was developed by White and collaborators [19,20]. It is based on Wilson's [21] phase-space cell approximation method, as a way to effectively to introduce the critical density fluctuations into a classical EoS. White's procedure was extended by Prausnitz and coworkers [22,23] and it has also been applied to several cubic [32, 35, 40], as well as non-cubic $\operatorname{EoS}[30-34,42,43]$. In this procedure, a recursive set of mathematical expressions is used to modify the free energy of fluids near the critical point. Therefore, it gives the correct non-analytical asymptotic behavior of fluids in the critical region [19,20,24,25].

This work focuses on the comparison of the capability of two crossover methods, specifically the ones developed by Kiselev and White, to describe properties of selected hydrocarbons, i.e. from methane to n-decane. In both cases, the Soave-Redlich-Kwong ( $S R K) E o S$ is used as the classical thermodynamic model. Besides, the two approaches have some advantages and disadvantages. Kiselev's method yields a closed-form analytical expression for the Helmholtz free energy and pressure, while White's procedure is solved numerically and requires an additional spline function for the representation of the thermodynamic surface of real fluids. The main disadvantage of Kiselev's approach is that it has several empirical system dependent parameters, which are not easily transferred to other compounds and mixtures [27]. On the other hand, White's method just has two additional parameters, transferable within the evaluated series. Furthermore, we have applied different parameterization procedures in an attempt to obtain reliable parameters for both approaches and to investigate the sensibility of the crossover parameters, as well as their dependence on the representation of the pure compound properties.

The rest of the paper is organized as follows. In section 2, we present a brief overview of the $S R K$ $E O S$ and describe the aforementioned crossover procedures. Section 3 is divided into three parts, in which the first one contains a discussion about the parameterization procedure, while the second and third parts are devoted to the study of the phase behavior and critical properties of n-alkanes. Section 4 presents the comparison of the EoS to investigate the phase behavior and critical properties of nalkanes (from methane to n-decane), and the prediction for heavy hydrocarbons (from n-dodecane to 
n-octadecane). Finally, in the last section, we summarize our findings and present the concluding remarks.

\section{Thermodynamic Models}

\subsection{Classical SRK equation of state}

The $S R K E O S$ is one of the most popular cubic thermodynamic models, due to its wide application in the refinery and gas-processing industries. Nonetheless, it is not as accurate as other cubic EoS, e.g. the Peng-Robinson $\operatorname{EoS}[26,27]$, in modeling the liquid densities of pure alkanes. This limitation can be improved by the use of a volume translation method or the modification of the input parameters to match the saturated properties far from the critical point. In this work, we briefly present the $S R K$ $E o S$, as it is the basis of the crossover models that will be later explained. Like other cubic thermodynamic models, the $S R K E O S$ can be explicitly defined in terms of pressure by the following equation [28]:

$P=\frac{R T}{v-b}-\frac{a(T)}{v(v+b)}$

where $a(T)$ is a temperature dependent constant of the attraction term expressed as:

$a(T)=a_{0}\left[1+k\left(1-\sqrt{T_{r}}\right)\right]^{2}$

in which $T_{r}=T / T_{c}$ is the reduced temperature and $k$ is a component specific parameter that can be expressed as a function of the acentric factor, $\omega[29]$ :

$k=0.480+1.574 \omega-0.175 \omega^{2}$

The constants $a_{0}$ and $b$ are related to the critical temperature and pressure. They are calculated by applying the criticality conditions, resulting in the following expressions:

$$
\begin{aligned}
& a_{0}=a\left(T_{c}\right)=\frac{0.4275 R^{2} T_{c}{ }^{2}}{P_{c}} \\
& b=\frac{0.08664 R T_{c}}{P_{c}}
\end{aligned}
$$

The total dimensionless Helmholtz free energy of the $S R K E O S$ is given by:

$$
\bar{A}(T, v)=\frac{A(T, v)}{R T}=-\ln (v-b)-\frac{a(T)}{R T b} \ln \left(\frac{v+b}{v}\right)
$$


Subsequently, to find the residual part of Helmholtz free energy, $\bar{A}^{\text {res }}$, the ideal part $\left(A^{\text {ideal }}=\right.$ $-R T \ln v$ ) should be subtracted from the total dimensionless Helmholtz free energy:

$\bar{A}^{r e s}(T, v)=\frac{A^{S R K}-A^{i d e a l}}{R T}=\ln \left(\frac{v}{v-b}\right)-\frac{a(T)}{R T b} \ln \left(\frac{v+b}{v}\right)$

\subsection{Crossover SRK equation of state}

\subsubsection{White's procedure}

White's method is implemented by transforming the grand canonical partition function into a functional integral [30-33]. In order to incorporate the long-range fluctuations into the free energy of a system calculated by a classical thermodynamic model, the interaction potential is divided into two main parts: a repulsive contribution $\left(u_{r e p}\right)$ and an attractive contribution $\left(u_{\text {att }}\right)$. The short-range fluctuations are entirely assigned to the repulsive term and the RGT transformations are only applied to the attractive part of the potential. The effect of the long wavelength contributions inferior to a given cut off length $L$ is calculated using a mean-field model and the short wavelength contributions are taken into account by the functional $\left(F_{S}\right)$ :

$F_{S}(\rho)=\int f_{s}(\rho) d r$

where $f_{s}$ is the Helmholtz free energy density for a homogeneous system with the molar density $\rho$. Since $F_{S}$ contains the fluctuations with short wavelengths, $f_{S}$ can be represented by a mean-field $E o S$. Here the importance of choosing the correct model is crucial for the representation of the system, as the procedure only significantly corrects the classical model close to the critical point [30]. In other words, the RGT procedure is considered to effectively account for the fluctuations close to the critical point. Hence, a suitable $E o S$ is required for precise predictions far from the critical region [34]. As a first approximation, the addition of the term $\alpha \rho^{2}$ is used to remove the attractive contributions [25]:

$f_{S}=f_{\text {classical EoS }}+\alpha \rho^{2}$

where $\alpha$ as an interaction volume is given by:

$\alpha=-\frac{1}{2} \int u_{a t t} d r$

in which $u_{a t t}$ is the attractive part of the pair potential. Following other works [32,35], the attractive contribution of the free energy density, i.e. $-\alpha \rho^{2}$, was approximated by the expression: 
$-\alpha \rho^{2} \approx-\frac{1}{2} a \rho^{2}$

where $a$ is the attractive term constant of the $S R K E O S$ calculated by Eq. (2).

In regions far from the critical point, the attractive term contribution can be correctly approximated by an analytical function; nonetheless, as the conditions of the system approach the critical one, a numerical procedure is required to estimate the attractive term constant $(\alpha)$. Similarly, the recursive procedure to incorporate density fluctuations is applied to $f_{s}$ and it is added to the saddle-point approximation, which gives the zero order solution of the grand partition function, yielding the total free energy density as given by:

$f=f_{n}-\alpha \rho^{2}$

where $f_{n}$ is the free energy density incorporating the density fluctuations on all wavelength scales.

The Helmholtz energy density of a system at a density $\rho$ can be described in a recursive manner as [36,37]:

$f_{n}(\rho)=f_{n-1}(\rho)+d f_{n}(\rho)$

where $d f_{n}$ corrects the previous free energy density $\left(f_{n-1}\right)$ by taking into account the density fluctuations through the following relation:

$d f_{n}(\rho)=-\left(\frac{k_{B} T}{2^{3 n} L^{3}}\right) \ln \frac{\Omega_{n}^{S}(\rho)}{\Omega_{n}^{l}(\rho)}$

Here, $\Omega^{s}$ and $\Omega^{l}$ are terms containing the density fluctuations for the short range and long range attraction, respectively, $k_{B}$ is the Boltzmann constant, $T$ is temperature and $L$ is a cutoff wavelength. The terms $\Omega_{n}^{s}$ and $\Omega_{n}^{l}$ are calculated through evaluation of the following integrals:

$\Omega_{n}^{\beta}(\rho)=\int_{0}^{\min \left(\rho, \rho_{\max }-\rho\right)} \exp \left[\frac{-G_{n}^{\beta}(\rho, x)}{K_{n}}\right] d x$

where $G_{n}^{\beta}$ is defined by the expression:

$G_{n}^{\beta}(\rho, x)=\frac{\bar{f}_{n}^{\beta}(\rho+x)-2 \bar{f}_{n}^{\beta}(\rho)+\bar{f}_{n}^{\beta}(\rho-x)}{2}$

and $\beta$ refers to both the short $(s)$ and long $(l)$ range attraction and $\bar{f}$ is given by:

$\bar{f}_{n}^{l}(\rho)=f_{n-1}(\rho)+\alpha \rho^{2}$ 
$\bar{f}_{n}^{S}(\rho)=f_{n-1}(\rho)+\alpha \rho^{2} \frac{\phi}{2^{2 n+1}}$

where $\phi$ is an adjustable parameter related to the initial shortest wavelength.

The subscript $n$ in Eq. (12) to (18) is the iteration number. In this work, five iterations were considered enough to correct the analytical behavior of the system in the critical region, as explained in previous work [35].

White's procedure adds two additional adjustable parameters $(L$ and $\phi)$ determined by the fitting crossover $E o S$ to experimental data, along with the critical parameters of the classical SRK EOS. The next section contains the details of the parameterization procedure.

Since classical cubic $E o S$ are normally expressed in terms of pressure, Eq. (18) shows the pressure of a system that accounts for the density contributions using White's procedure:

$$
P_{n}=P+\rho \frac{\partial d f_{n}(\rho)}{\partial \rho}-d f_{n}(\rho)
$$

It is important to notice that, in this work, the term $P$ in the previous expression is obtained with Eq. (1). Besides, the terms $\frac{\partial d f_{n}(\rho)}{\partial \rho}$ and $d f_{n}(\rho)$ are estimated with a spline function, as mentioned previously, using 500 density points, from $\rho=10^{-12}$ to $\rho_{\max }=\frac{1}{0.999 b}$, to avoid numerical issues.

\subsubsection{Kiselev's procedure}

Kiselev [7,8] developed a method for incorporating the scaling laws into a classical analytical EoS. The author suggested separating the Helmholtz free energy into a critical part, $\Delta A$, which is affected by critical fluctuations and an analytic background part unaffected by them. Consequently, the expression for the dimensionless free energy is given by:

$$
\bar{A}(T, v)=\frac{A(T, v)}{R T}=\Delta \bar{A}(\Delta T, \Delta v)-\frac{v}{v_{0 c}} \bar{P}_{0}(T)+\bar{\mu}_{0}(T)
$$

where $\bar{P}_{0}(T)=P\left(T, v_{0 c}\right) v_{0 c} / R T$ is the dimensionless pressure at $v=v_{0 c}$. The dimensionless deviations of the critical temperature and volume are $\Delta T=\left(T-T_{0 c}\right) / T_{0 c}$ and $\Delta v=\left(v-v_{0 c}\right) / v_{0 c}$, respectively. Finally, $\bar{\mu}_{0}(T)$ is the dimenssionless analytic function of temperature and it is derived from the condition $\Delta \bar{A}(\Delta T, \Delta v=0)=0$, resulting in the expression given by: 
$\bar{\mu}_{0}(T)=\bar{A}^{r e s}\left(T, v_{0 c}\right)+\bar{A}_{0}(T)-\ln v_{0 c}+\bar{P}_{0}(T)$

in which $\bar{A}_{0}(T)$ is the dimensionless temperature dependent part of Helmholtz free energy for an ideal gas. The detail of derivations are presented in the supplementary material.

Since any classical equation of state does not reproduce the thermodynamic surface of a fluid in the critical region, the critical part of Helmholtz free energy $(\Delta A)$ re-expressed from a function of the renormalized $\Delta T$ and $\Delta v$ to the real critical parameters of the system: $\tau=T / T_{c}-1$ and $\eta=v / v_{c}-$ 1 , respectively. In order to take into account the fluctuations induced close to the critical point and the difference between the classical critical parameters $\left(T_{0 c}\right.$ and $\left.v_{0 c}\right)$, and the real critical parameters $\left(T_{c}\right.$ and $\left.v_{c}\right)$, Kiselev et al. [7-9] introduced the renormalized value of temperature and volume together with the critical shifts of temperature and volume as described by the following equations:

$\bar{\tau}=\tau Y(q)^{(-\alpha / 2 \Delta)}+(1+\tau) \Delta \tau_{c} Y(q)^{((2(2-\alpha)) / 3 \Delta)}$

$\bar{\eta}=\eta Y(q)^{((\gamma-2 \beta) / 4 \Delta)}+(1+\eta) \Delta \eta_{c} Y(q)^{((2-\alpha) / 2 \Delta)}$

where $\Delta \tau_{c}=\left(T_{c} / T_{0 c}-1\right)$, and $\Delta \eta_{c}=\left(v_{c} / v_{0 c}-1\right)$ are the dimensionless shifts of the critical parameters and $\gamma=1.24, \beta=0.325, \alpha=2-\gamma-2 \beta=0.110$ and $\Delta=0.51$ are the universal nonclassical critical exponents in the scaling power laws. These critical exponents characterize the nonanalytical behavior of thermodynamic functions in the vicinity of the critical point, such as order parameter, heat capacity, isothermal compressibility and other properties $[4,38]$.

The crossover function $Y(q)$ in Eq. (22) and (23) is related to the distance from the critical point, which can be defined as:

$Y(q)=\left(\frac{q}{1+q}\right)^{2 \Delta}$

The function $Y(q)$ approaches unity far from the critical point, as the value of $q$ tends to infinity $(q \rightarrow \infty)$, while near the critical point it vanishes as $q^{2 \Delta}$, since tends $q$ to zero $(q \rightarrow 0)$. Thus, the parametric variable $q$ is a renormalized measure of the distance from the critical point and is calculated iteratively from the parametric model, given by:

$$
\left(q^{2}-\frac{\tau}{G i}\right)\left[1-\frac{p^{2}}{4 b^{2}}\left(1-\frac{\tau}{q^{2} G i}\right)\right]=\left\{\frac{b f(\eta, \tau)}{m_{0} G i \beta}\right\}^{2} Y^{(1-2 \beta) / \Delta}
$$


The empirical function $f(\eta, \tau)$ is used to eliminate the symmetry with respect to the order parameter $(\eta)$, and in this work was considered equal to:

$f(\eta, \tau)=\eta\left(1+v_{1} e^{-10 \eta}\right)+d_{1} \tau$

where $v_{1}$ and $d_{1}$ are system-dependent parameters. The term proportional to $d_{1} \tau$ in Eq. (26) corresponds to a projection of the rectilinear diameter of the coexistence curve in the temperature density variables $\rho_{d}=\left(\rho_{G}+\rho_{L}\right) / 2=\rho_{c}\left(1+d_{1} \tau\right)$, while the term proportional to $v_{1} \exp (-10 \eta)$ takes into account the asymmetry of $Y(q)$ with respect to a transformation $\eta \rightarrow-\eta$, which ensures that $Y \rightarrow 1$ at $v \ll v_{c}$, hence, ensuring that the crossover is complete. The parameter $m_{0}$ is considered as either universal or system dependent parameter. As a result, a total of four crossover parameters $\left(m_{0}, G i, d_{1}\right.$ and $\left.v_{1}\right)$ can be treated as compound specific.

The complete transformation of the classical Helmholtz energy into the crossover form is done via the following steps:

1. Define the critical part of Helmholtz free energy, $\Delta \bar{A}$, as given by:

$\Delta \bar{A}(\Delta T, \Delta v)=\bar{A}^{r e s}(T, v)-\bar{A}^{r e s}\left(T, v_{0 c}\right)+\Delta v \bar{P}_{0}(T)-\ln (\Delta v+1)$

In addition, the representation of the Ising-like behavior asymptotically close to the critical point requires the replacement of the classical dimensionless temperature, $\Delta T$, and volume, $\Delta v$, with the renormalized values $\bar{\tau}$ and $\bar{\eta}$; as well as the classical properties, $T$ and $v$, with their rescaled values $\left(\bar{T}=T_{0 c}(1+\bar{\tau})\right.$ and $\left.\bar{v}=v_{0 c}(1+\bar{\eta})\right)$ :

$\Delta \bar{A}(\bar{\tau}, \bar{\eta})=\bar{A}^{r e s}(\bar{T}, \bar{v})-\bar{A}^{r e s}\left(\bar{T}, v_{0 c}\right)+\bar{\eta} \bar{P}_{0}(\bar{T})-\ln (\bar{\eta}+1)$

2. Determine the background part of Helmholtz energy, as expressed by:

$\bar{A}^{r e s}(\bar{T}, \bar{v})-\Delta \bar{A}(\bar{\tau}, \bar{\eta})=\bar{A}^{r e s}\left(T, v_{0 c}\right)-\Delta v \bar{P}_{0}(T)-\ln v_{0 c}+\bar{A}_{0}(T)$

3. Finally, the renormalized total Helmholtz energy is given as a sum of the critical part at the rescaled variables $\bar{\tau}$ and $\bar{\eta}$, and the background part:

$\bar{A}(T, v)=\bar{A}^{r e s}(\bar{T}, \bar{v})-\bar{A}^{r e s}\left(\bar{T}, v_{0 c}\right)+\bar{\eta} \bar{P}_{0}(\bar{T})-\ln (\bar{\eta}+1)+\bar{A}^{r e s}\left(T, v_{0 c}\right)-\Delta v \bar{P}_{0}(T)-\ln v_{0 c}+$ $\overline{A_{0}}(T)$

The final crossover cubic equation of state in this case reads: 
$P=-\left(\frac{\partial A}{\partial v}\right)_{T}=-\frac{R \bar{T}}{v_{c}}\left(\frac{\partial \Delta \bar{A}(\bar{\tau}, \bar{\eta})}{\partial \eta}\right)+\bar{P}_{0}(T)$

The detailed expressions are given in supplementary material.

\section{Application to fluids}

In this work, we have applied the two aforementioned approaches of the crossover $S R K E O S$ to describe the saturated and critical properties of pure n-alkanes (from methane to n-decane), as well as isothermal pressure-density curves. The first approach involved the application of White's numerical procedure for improving the classical model far away and close to the critical point, while the second one followed Kiselev's approach.

The critical properties and acentric factor values of the studied pure compounds are listed in Table 1. The reported properties were taken from the NIST database [39].

In order to compare the two models, $P V T$ data was taken from the NIST database [39] along the critical isotherm and along a temperature above $T_{c}$ and the pressure steps are up to $3 P_{c}$. The temperature above critical was the integer-multiple of $50 \mathrm{~K}$ which was more than $35 \mathrm{~K}$ above $T_{c}$, except for the cases where the highest temperature did not match this procedure, e.g. the maximum temperature for isothermal $P V T$ data for $\mathrm{C}_{9}$ is $575 \mathrm{~K}$, which is below the critical temperature of the component. This procedure is similar to the one developed by Janeček et al. [27].

Table 1. The critical properties and acentric factor values of studied pure compounds. The reported properties are taken from NIST [39].

\begin{tabular}{|c|c|c|c|c|}
\hline \multirow{2}{*}{ Comp. } & \multicolumn{3}{|c|}{ Critical Properties } & Acentric factor \\
\cline { 2 - 5 } & $T_{c}(\mathrm{~K})$ & $P_{c}($ bar $)$ & $v_{c}\left(\mathrm{~cm}^{3} / \mathrm{mol}\right)$ & $\omega(-)$ \\
\hline C1 & 190.6 & 45.99 & 98.60 & 0.0115 \\
\hline C2 & 305.3 & 48.72 & 145.8 & 0.0994 \\
\hline C3 & 369.9 & 42.51 & 200.0 & 0.1523 \\
\hline C4 & 425.1 & 37.96 & 254.9 & 0.2002 \\
\hline C5 & 469.7 & 33.70 & 311.0 & 0.2515 \\
\hline C6 & 507.8 & 30.34 & 369.6 & 0.3013 \\
\hline C7 & 540.1 & 27.36 & 431.9 & 0.3495 \\
\hline C8 & 569.3 & 24.97 & 486.3 & 0.3995 \\
\hline C9 & 594.5 & 22.81 & 552.5 & 0.4435 \\
\hline C10 & 617.7 & 21.03 & 609.8 & 0.4923 \\
\hline
\end{tabular}


Since the parameters $a_{0}$ and $b$ in Eqs. (4) and (5) are related to the critical temperature and pressure, they are calculated with the experimental $T_{c}$ and $P_{c}$ for Kiselev's procedure (Table 1). On the other hand, in the case of White's procedure, they are modified by the classical critical parameters (Table 2) in order to improve the description of the properties far away from the critical point.

\subsection{Adjustment of parameters}

For a pure fluid, the crossover $E o S$ on the basis of Kiselev's procedure has three sets of system dependent parameters: (1) the crossover parameters $m_{0}, G i, d_{1}$ and $v_{1},(2)$ the critical shifts $\Delta \tau_{c}$ and $\Delta \eta_{c}$, (3) and the classical parameters: $T_{0 c}, P_{0 c}, v_{0 c}$ and $\omega$. In practice, the classical critical parameters are different from the experimental critical parameters: $T_{c}, v_{c}$ and $P_{c}$. Nevertheless, the critical temperature and pressure shifts did not bring a significant improvement in the representations of the properties of the fluids. Consequently, $T_{0 c}$ and $P_{0 c}$ are considered equal to $T_{c}$ and $P_{c}$, respectively, and the critical volume is increased slightly to improve the representation of the experimental data. In this work, $m_{0}$ is kept equal to 1 . As a result, the crossover $E o S$ using Kiselev's procedure contains five adjustable pure component parameters, three from the crossover part $\left(G i, d_{1}\right.$ and $\left.v_{1}\right)$ and two from the classical part $\left(v_{0 c}\right.$ and $\left.\omega\right)$. One of the main objectives of this work is to investigate the effect of the crossover parameters on the accuracy of $V L E$ and PVT predictions and to reduce the total number of system dependent adjustable parameters. Therefore, we considered three methods, in which further restrictions are introduced, reducing the number of free adjustable parameters from 5 , in the first method, to three, in the third one.

In the case of the crossover EoS using White's procedure, two additional crossover parameters are introduced in the classical thermodynamic model. The first one is the cut-off length $(L)$, while the second is $\phi$, which is a function of the initial shortest wavelength of the density fluctuations [24]. Besides, instead of the utilization of the critical shifts for the correction of the critical point, the classical critical pressure, temperature and acentric factor $\left(T_{0 c}, P_{0 c}\right.$ and $\left.\omega_{0}\right)$ are used in White's crossover method. The parameters are estimated via the fitting of the saturated pressure and volumes far away from the critical point, e.g. from $T_{r}=0.5$ to 0.75 . This procedure causes an overestimation of the critical point that is corrected with the renormalization procedure described in the previous section, allowing the precise representation of the experimental saturated data and of the correct shape of the coexistence curve close to the critical point [3]. Two parameterization methods were tested. In the first method, the value of $\phi$ was kept constant, while the four other adjustable parameters were 
used to fit the critical and saturated experimental data. In the second method, both the crossover and classical parameters were optimized in order to minimize the objective function containing the calculated and experimental properties.

The objective function used in all methods is given by:

$$
\begin{gathered}
\text { O.F. }=\sum\left(\frac{P_{c a l c}^{\text {sat }}-P_{\text {exp }}^{\text {sat }}}{P_{\text {exp }}^{\text {sat }}}\right)^{2}+\sum\left(\frac{\rho_{\text {calc }}^{l}-\rho_{\text {exp }}^{l}}{\rho_{\text {exp }}^{l}}\right)^{2}+\sum\left(\frac{\rho_{\text {calc }}^{v}-\rho_{\text {exp }}^{v}}{\rho_{\text {exp }}^{v}}\right)^{2}+\sum\left(\frac{P_{c a l c}^{\text {isot }}-P_{\text {exp }}^{\text {isot }}}{P_{\text {exp }}^{\text {ist }}}\right)^{2}+ \\
\left(\frac{P_{c, \text { calc }}-P_{c, \text { exp }}}{P_{c, \text { exp }}}\right)^{2}+\left(\frac{T_{c, \text { calc }}-T_{c, \text { exp }}}{T_{c, \text { exp }}}\right)^{2}+\left(\frac{\rho_{c, \text { calc }}-\rho_{c, \text { exp }}}{\rho_{c, \text { exp }}}\right)^{2}
\end{gathered}
$$

\subsection{White's procedure}

\subsubsection{Method I}

As mentioned previously, the crossover $S R K E O S$ using White's recursive procedure contains 5 parameters for each component in a system. Three parameters are related to the classical term, which are the classical critical pressure, temperature and acentric factor. The last two parameters are related to the crossover term and represent the cut-off length $(L)$ and initial shortest wavelength $(\phi)$. These two crossover parameters are strongly correlated. In Method I, we deliberately set $\phi$ to a constant value $(\phi=2)$ to remove the influence of the strong correlation. Then the rest of the parameters were obtained by minimizing Eq. (32). The initial values for the classical critical parameters were obtained from the regression of the classical model in a reduced temperature range of 0.5 to 0.75 .

The first columns of Table 2 show the optimum parameters using the first method of White's procedure. In order to observe a trend in the parameter values, they were plotted against the molecular weight of the hydrocarbons (Figs. 1 and 2). In Fig. 1, it is seen that the classical critical parameters follow a similar trend to the experimental values (Table 1). The main difference is that the critical temperatures and pressures are overestimated to improve the description of the phase densities far away from the critical region [32]. This procedure was also done for other cubic crossover $\operatorname{EoS}$ [32, $35,40]$ and requires the modification of the experimental acentric factors for the correct representation of the vapor pressures of the pure fluids. In fact, the comparison of Tables 1 and 2 shows that $\omega$, in the crossover model, are smaller than the experimental values, and, in the case of methane, it becomes negative. Negative acentric factor values are also observed for molecules like hydrogen and helium [41]. The renormalization group method developed by White and coworkers 
corrects the classical model in the critical region, yielding a precise representation of the phase envelope, giving the correct shape of the coexistence diagram. Finally, the comparison of the parameters estimated in this works with other publications $[32,35]$ indicates the values are similar to the ones defined for other implementations of the crossover SRKEOS to describe the properties of nalkanes.

Table 2. The values of adjusted parameters in the crossover SRK EOS using the two methods of White's procedure for the selected n-alkanes.

\begin{tabular}{|c|c|c|c|c|c|c|c|c|c|}
\hline \multirow{2}{*}{ Comp. } & \multicolumn{4}{|c|}{ Method I } & \multicolumn{5}{|c|}{ Method II } \\
\hline & $T_{0 c}(\mathrm{~K})$ & $P_{0 c}$ (bar) & $\omega_{0}(-)$ & $L\left(\mathrm{~A}^{\circ}\right)$ & $T_{0 c}(\mathrm{~K})$ & $P_{0 c}$ (bar) & $\omega_{0}(-)$ & $L\left(\mathrm{~A}^{\circ}\right)$ & $\phi(-)$ \\
\hline $\mathrm{C}_{1}$ & 197.7 & 49.88 & -0.049 & 4.390 & 197.7 & 49.88 & -0.049 & 4.390 & 2.000 \\
\hline $\mathrm{C}_{2}$ & 317.1 & 54.75 & 0.033 & 4.800 & 317.1 & 54.75 & 0.033 & 4.800 & 2.000 \\
\hline $\mathrm{C}_{3}$ & 383.4 & 47.75 & 0.087 & 5.338 & 383.3 & 47.65 & 0.086 & 5.468 & 1.750 \\
\hline $\mathrm{C}_{4}$ & 440.0 & 43.05 & 0.137 & 5.721 & 441.4 & 43.00 & 0.128 & 5.864 & 1.750 \\
\hline $\mathrm{C}_{5}$ & 486.8 & 38.71 & 0.182 & 6.045 & 488.0 & 38.63 & 0.174 & 6.212 & 1.750 \\
\hline $\mathrm{C}_{6}$ & 525.2 & 35.27 & 0.234 & 6.296 & 527.1 & 35.08 & 0.221 & 6.506 & 1.750 \\
\hline $\mathrm{C}_{7}$ & 559.7 & 32.30 & 0.276 & 6.548 & 560.5 & 32.10 & 0.271 & 6.922 & 1.500 \\
\hline $\mathrm{C}_{8}$ & 589.9 & 29.81 & 0.320 & 6.817 & 591.0 & 29.83 & 0.315 & 7.084 & 1.500 \\
\hline $\mathrm{C}_{9}$ & 616.5 & 27.61 & 0.364 & 7.092 & 618.4 & 27.58 & 0.354 & 7.354 & 1.500 \\
\hline $\mathrm{C}_{10}$ & 639.8 & 25.71 & 0.410 & 7.338 & 639.4 & 25.50 & 0.411 & 7.603 & 1.500 \\
\hline
\end{tabular}
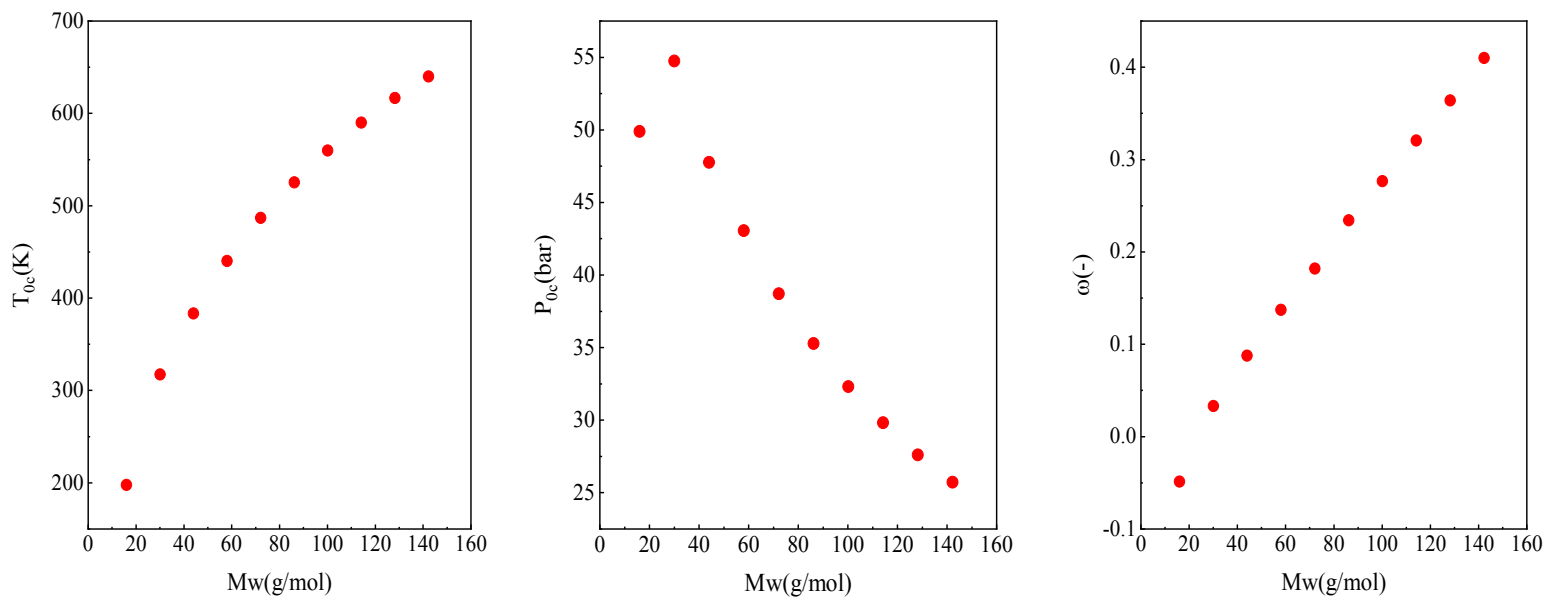

Fig. 1. Classical critical parameters for the crossover $S R K E O S$ using the first approach of White's procedure as a function of the molecular weight for selected n-alkanes. 


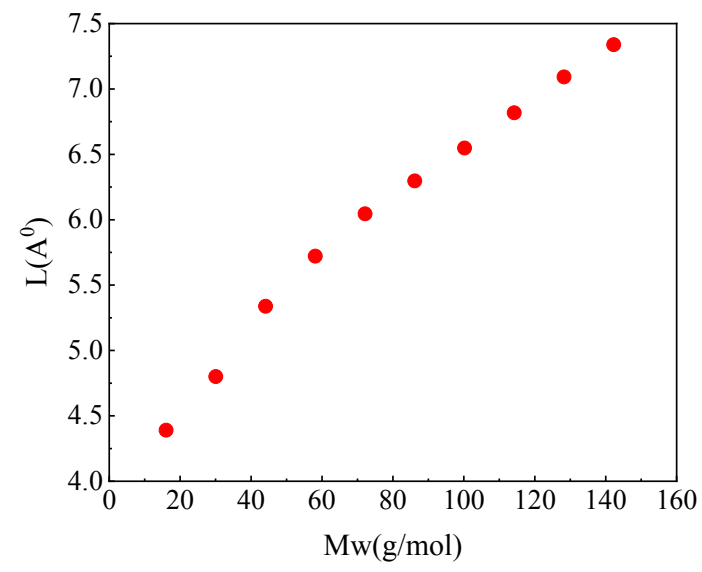

Fig. 2. Cut-off length for the crossover $S R K E O S$ using the first approach of White's procedure as a function of the molecular weight for selected n-alkanes.

After the estimation of the best parameters using the first approach of the recursive procedure, the model was applied to the calculation of the saturated and critical properties, along with a critical and supercritical isotherm. The first columns of Table 3 show the deviations of the saturated properties and one critical and a supercritical isotherm for the selected n-alkanes studied in this work. The deviations indicate that the model is capable of correctly describing the saturated properties. Although the model yields an inferior description of the isothermal pressure-density curves in comparison to representation of the saturated properties, the results are much superior to the cubic model, which gives an overall average absolute deviation (AAD) of 53\% (Table 10). The final comparison of the calculated and experimental data is given in the first columns of Table 4 . This table shows the AAD\% of the critical properties, where it is seen that the model correctly predicts the critical temperatures and pressures of the system; however, the deviations in terms of the critical pressure and volume increase substantially with the molecular weight of the hydrocarbon. In order to improve this behavior, a second approach was applied, in which the crossover parameter $\phi$ was also utilized as an adjustable parameter. 
Table 3. AAD $\%$ of the calculated and experimental saturated and isotherms of selected n-alkanes using the two methods for White's procedure.

\begin{tabular}{|c|c|c|c|c|c|c|c|c|}
\hline \multirow{2}{*}{ Comp. } & \multicolumn{4}{|c|}{ Method I } & \multicolumn{4}{|c|}{ Method II } \\
\hline & $\Delta \mathrm{P}^{\mathrm{sat}}(\%)$ & $\Delta \rho^{\operatorname{liq}}(\%)$ & $\Delta \rho^{\text {vap }}(\%)$ & $\Delta \mathrm{P}^{\text {isot }}(\%)$ & $\Delta \mathrm{P}^{\mathrm{sat}}(\%)$ & $\Delta \rho^{\text {liq }}(\%)$ & $\Delta \rho^{\text {vap }}(\%)$ & $\Delta \mathrm{P}^{\mathrm{isot}(\%)}$ \\
\hline$\overline{\mathrm{C}_{1}}$ & 3.63 & 3.23 & 1.90 & 3.73 & 3.63 & 3.23 & 1.90 & 3.73 \\
\hline $\mathrm{C}_{2}$ & 2.11 & 3.29 & 2.33 & 4.49 & 2.11 & 3.29 & 2.33 & 4.49 \\
\hline $\mathrm{C}_{3}$ & 2.34 & 2.66 & 2.18 & 5.07 & 2.12 & 2.72 & 3.25 & 5.27 \\
\hline $\mathrm{C}_{4}$ & 1.91 & 2.66 & 2.27 & 5.37 & 2.64 & 2.26 & 2.21 & 5.09 \\
\hline $\mathrm{C}_{5}$ & 2.05 & 2.17 & 1.73 & 6.05 & 2.76 & 1.89 & 1.94 & 5.83 \\
\hline $\mathrm{C}_{6}$ & 1.68 & 2.36 & 2.23 & 7.30 & 2.61 & 1.91 & 1.99 & 6.74 \\
\hline $\mathrm{C}_{7}$ & 1.90 & 1.74 & 1.39 & 7.50 & 2.47 & 2.19 & 2.33 & 7.51 \\
\hline $\mathrm{C}_{8}$ & 2.02 & 1.52 & 1.38 & 8.30 & 2.43 & 1.77 & 2.71 & 8.50 \\
\hline $\mathrm{C}_{9}$ & 1.85 & 1.80 & 1.16 & 15.3 & 2.61 & 2.30 & 1.97 & 15.28 \\
\hline $\mathrm{C}_{10}$ & 1.85 & 1.53 & 0.94 & 9.34 & 2.04 & 4.02 & 2.72 & 9.61 \\
\hline
\end{tabular}

*Average Absolute Deviation $(\mathrm{AAD} \%)=(1 / n)\left(\sum\left|X^{\text {calc }}-X^{\text {exp }}\right| / X^{\text {exp }}\right) * 100$

\subsubsection{Method II}

In an attempt to improve the description of the critical pressure and volume for the larger hydrocarbons, a different procedure for the optimization of the parameters was applied. Initially, the 5 parameters of the crossover $S R K$ using the recursive procedure were regressed simultaneously; nevertheless, due to the strong correlation between the parameters, the deviations regarding the critical properties were not reduced. Hence, a modification was proposed, instead of parameterizing the five values at the same time, different values of $\phi$ were considered constant and the rest of the parameters were adjusted in other to minimize Eq. (32). This method led to the improvement of the representation of the critical points, especially for the heavier hydrocarbons, without changing the other parameters substantially.

In principle, $\phi$ can be determined from a low density microscopic calculation [25]; however, it is usually treated in a phenomenological matter. This means that this parameter is fitted to the pure component properties. In fact, several crossover $E o S$ use an increasing linear correlation with respect to the molecular weight for the alkane homologous series [30, 42, 43]; nevertheless, in Method II, smaller values for $\phi$ resulted in better critical volume representations of heavier hydrocarbons.

Table 2 shows the final values of the parameters of the crossover model using the second method for White's procedure. It can be observed that, in addition to the similarity of the values between the previous method, the trends are also alike. 
Similarly, the values of the parameters were used to calculate the saturated properties and the isothermal curves (Table 3). The comparison of the AAD\% with the previous method indicates that the second method slightly increases the deviations, although it is still capable of correctly correlating the properties. On the other hand, the second method improves substantially the description of the critical properties (Table 4).

Table 4. AAD $\%$ of the calculated and experimental critical properties of selected n-alkanes using the two methods for White's procedure.

\begin{tabular}{|ccccccccc|}
\hline \multirow{2}{*}{ Comp. } & \multicolumn{3}{c}{ Method I } & & \multicolumn{3}{c|}{ Method II } \\
\cline { 2 - 4 } \cline { 6 - 8 } \cline { 6 - 8 } & $\Delta T_{c}(\%)$ & $\Delta P_{c}(\%)$ & $\Delta \rho_{c}(\%)$ & & $\Delta T_{c}(\%)$ & $\Delta P_{c}(\%)$ & $\Delta \rho_{c}(\%)$ \\
\hline $\mathrm{C}_{1}$ & 0.15 & 0.62 & 1.38 & & 0.15 & 0.62 & 1.38 \\
$\mathrm{C}_{2}$ & 0.02 & 0.01 & 0.00 & & 0.02 & 0.01 & 0.00 \\
$\mathrm{C}_{3}$ & 0.05 & 0.02 & 0.89 & & 0.18 & 0.02 & 0.05 \\
$\mathrm{C}_{4}$ & 0.20 & 0.01 & 0.82 & & 0.04 & 0.04 & 0.04 \\
$\mathrm{C}_{5}$ & 0.13 & 0.02 & 1.85 & & 0.01 & 0.03 & 0.85 \\
$\mathrm{C}_{6}$ & 0.41 & 0.58 & 1.99 & & 0.13 & 0.53 & 0.98 \\
$\mathrm{C}_{7}$ & 0.24 & 0.33 & 2.18 & & 0.28 & 0.43 & 0.92 \\
$\mathrm{C}_{8}$ & 0.17 & 0.93 & 3.85 & & 0.43 & 0.34 & 0.11 \\
$\mathrm{C}_{9}$ & 0.00 & 2.38 & 3.22 & & 0.18 & 0.40 & 0.34 \\
$\mathrm{C}_{10}$ & 0.01 & 3.44 & 4.34 & & 0.56 & 0.40 & 1.05 \\
\hline
\end{tabular}

\subsection{Kiselev's procedure}

\subsubsection{Method I}

In the first step, the five parameters were optimized simultaneously by minimizing the objective function defined in Eq. (32). In this method, three parameters are related to the critical part (Gi, $d_{1}$ and $\left.v_{1}\right)$ and two stem from the classical part $\left(v_{0 c}\right.$ and $\left.\omega\right)$. The results of this optimization are reported in Table 5. The AAD\% of saturated properties and isothermal pressure-density are listed in the first columns of Table 6. Generally, large deviations are found for the vapor density of hydrocarbons with lighter molecular weight, while the liquid phase volume representation is more accurate.

Table 9 shows the deviation of critical pressures for the selected hydrocarbons calculated by Kiselev's procedure compared to the experimental values. Kiselev's procedure corrects the representation of the critical properties and precisely describes the critical properties. 
In this method, it was not possible to define a simple correlation between the crossover parameters and the molecular weight. As it is seen in Fig. 3, the values of the parameters are scattered along the axis describing the molecular weight of the hydrocarbons.

The influence of crossover and classical parameters on the representation of the saturated properties and isothermal pressure is represented in Fig. 4. In this figure, different values of $v_{1}$ and $d_{1}$ were applied in the plot of the two isotherms and saturated pressure of n-hexane. The comparison of the results with experimental data indicates that the effect of $v_{1}$ is much greater than $d_{1}$ in the prediction of the thermodynamic properties of the pure component. For this reason, in the next step, we have tried to reduce the number of adjustment crossover parameters.

$\mathbf{S}$
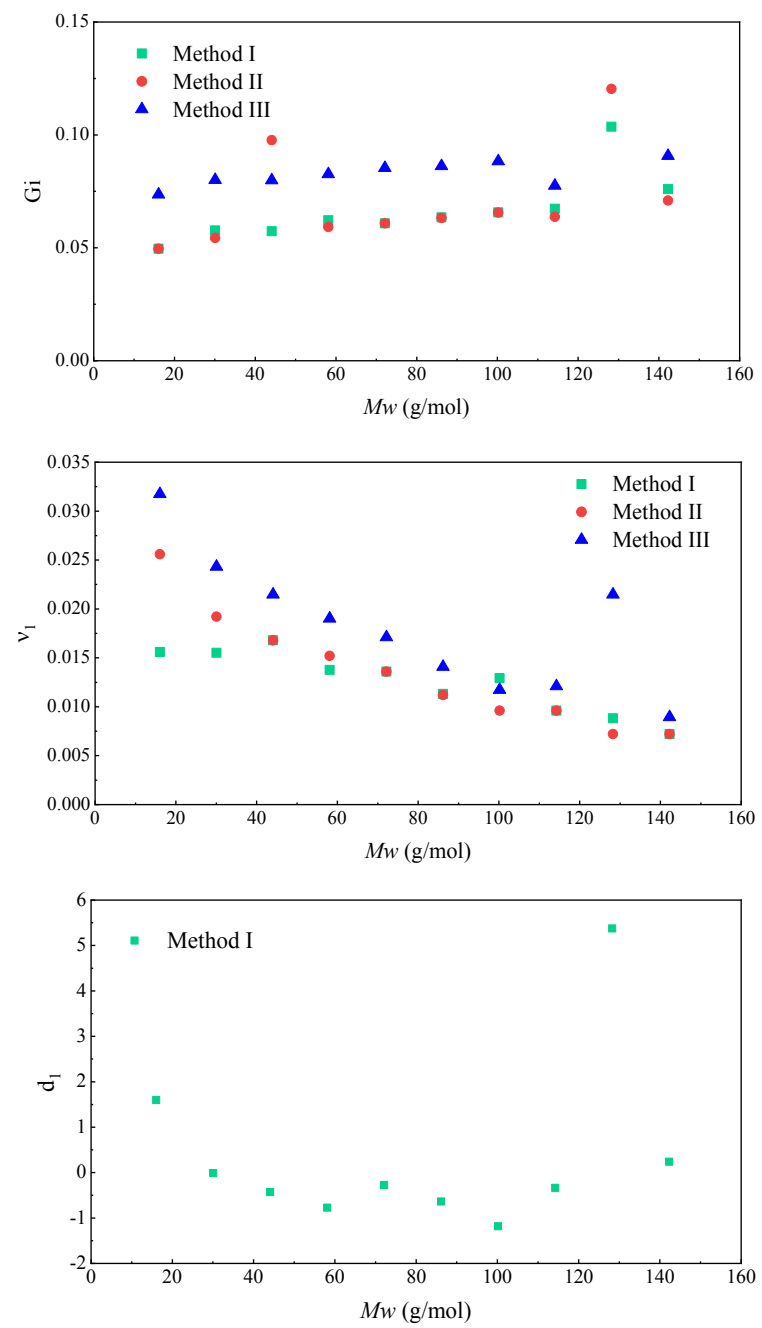

Fig. 3. Optimized Gi, $d_{1}$, and $v_{1}$ values for the crossover SRK EoS using different methods of Kiselev's procedure as a function of the molecular weight for selected n-alkanes. 
Table 5. The values of adjusted parameters in the crossover SRK EOS using the first method of Kiselev's procedure for selected n-alkanes.

\begin{tabular}{|c|c|c|c|c|c|}
\hline Comp. & $\begin{array}{c}\mathrm{v}_{0 \mathrm{c}} \\
\left(\mathrm{cm}^{3} / \mathrm{mol}\right)\end{array}$ & $\omega(-)$ & $\mathrm{Gi}(-)$ & $\mathrm{d}_{1}(-)$ & $\mathrm{v}_{1}(-)$ \\
\hline $\mathrm{C}_{1}$ & 115.703 & 0.013 & 0.050 & 1.597 & 0.016 \\
\hline $\mathrm{C}_{2}$ & 181.589 & 0.110 & 0.058 & -0.013 & 0.016 \\
\hline $\mathrm{C}_{3}$ & 263.969 & 0.169 & 0.057 & -0.430 & 0.017 \\
\hline $\mathrm{C}_{4}$ & 336.084 & 0.219 & 0.062 & -0.773 & 0.014 \\
\hline $\mathrm{C}_{5}$ & 433.135 & 0.273 & 0.061 & -0.273 & 0.014 \\
\hline $\mathrm{C}_{6}$ & 520.797 & 0.322 & 0.063 & -0.636 & 0.011 \\
\hline $\mathrm{C}_{7}$ & 664.123 & 0.380 & 0.066 & -1.185 & 0.013 \\
\hline $\mathrm{C}_{8}$ & 698.791 & 0.424 & 0.067 & -0.337 & 0.010 \\
\hline $\mathrm{C}_{9}$ & 799.575 & 0.484 & 0.104 & 5.374 & 0.009 \\
\hline $\mathrm{C}_{10}$ & 875.325 & 0.519 & 0.076 & 0.239 & 0.007 \\
\hline
\end{tabular}

Table 6. AAD $\%$ of the calculated and experimental saturated and isotherms of selected n-alkanes using the three methods for Kiselev's procedure.

\begin{tabular}{|c|c|c|c|c|c|c|c|c|c|c|c|c|}
\hline \multirow[b]{2}{*}{ Comp. } & \multicolumn{4}{|c|}{ Method I } & \multicolumn{4}{|c|}{ Method II } & \multicolumn{4}{|c|}{ Method III } \\
\hline & $\begin{array}{c}\Delta \mathrm{P}^{\text {sat }} \\
(\%)\end{array}$ & $\begin{array}{l}\Delta \rho^{\text {liq }} \\
(\%)\end{array}$ & $\begin{array}{c}\Delta \rho^{\text {vap }} \\
(\%)\end{array}$ & $\begin{array}{c}\Delta \mathrm{P}^{\text {isot }} \\
(\%)\end{array}$ & $\begin{array}{c}\mathrm{P}^{\text {sat }} \\
(\%)\end{array}$ & $\begin{array}{l}\Delta \rho^{\text {liq }} \\
(\%)\end{array}$ & $\begin{array}{c}\Delta \rho^{\text {vap }} \\
(\%)\end{array}$ & $\begin{array}{c}\Delta \mathrm{P}^{\text {isot }} \\
(\%)\end{array}$ & $\begin{array}{c}\Delta \mathrm{P}^{\text {sat }} \\
(\%)\end{array}$ & $\begin{array}{l}\Delta \rho^{\text {liq }} \\
(\%)\end{array}$ & $\begin{array}{c}\Delta \rho^{\text {vap }} \\
(\%)\end{array}$ & $\begin{array}{c}\Delta \mathrm{P}^{\text {isot }} \\
(\%)\end{array}$ \\
\hline $\mathrm{C}_{1}$ & 1.83 & 2.01 & 2.73 & 1.35 & 1.44 & 2.23 & 3.67 & 1.95 & 1.74 & 1.39 & 3.28 & 4.69 \\
\hline $\mathrm{C}_{2}$ & 1.73 & 1.45 & 2.36 & 1.17 & 1.52 & 1.29 & 2.68 & 1.75 & 1.61 & 1.03 & 3.06 & 5.19 \\
\hline $\mathrm{C}_{3}$ & 1.49 & 1.06 & 2.61 & 1.57 & 1.81 & 0.91 & 2.99 & 1.40 & 1.48 & 1.10 & 3.24 & 4.88 \\
\hline $\mathrm{C}_{4}$ & 1.91 & 1.20 & 2.19 & 0.05 & 1.74 & 0.93 & 2.45 & 1.73 & 1.60 & 1.77 & 3.36 & 5.14 \\
\hline $\mathrm{C}_{5}$ & 1.57 & 1.36 & 2.50 & 0.13 & 1.57 & 1.33 & 2.54 & 1.14 & 1.48 & 2.87 & 3.49 & 7.59 \\
\hline $\mathrm{C}_{6}$ & 1.52 & 1.71 & 2.17 & 0.98 & 1.55 & 1.61 & 2.21 & 0.95 & 1.39 & 2.47 & 3.15 & 5.80 \\
\hline $\mathrm{C}_{7}$ & 1.30 & 2.41 & 1.81 & 2.07 & 1.59 & 2.03 & 1.88 & 1.23 & 1.46 & 3.09 & 2.77 & 4.88 \\
\hline $\mathrm{C}_{8}$ & 1.59 & 2.47 & 2.03 & 1.43 & 1.61 & 2.58 & 1.88 & 1.45 & 1.59 & 3.42 & 2.64 & 4.65 \\
\hline $\mathrm{C}_{9}$ & 2.22 & 4.45 & 3.31 & 1.88 & 2.46 & 2.11 & 2.84 & 2.62 & 2.93 & 3.89 & 4.88 & 14.04 \\
\hline $\mathrm{C}_{10}$ & 2.03 & 2.96 & 2.03 & 0.84 & 1.89 & 2.93 & 2.05 & 1.11 & 1.61 & 3.73 & 3.12 & 5.27 \\
\hline
\end{tabular}
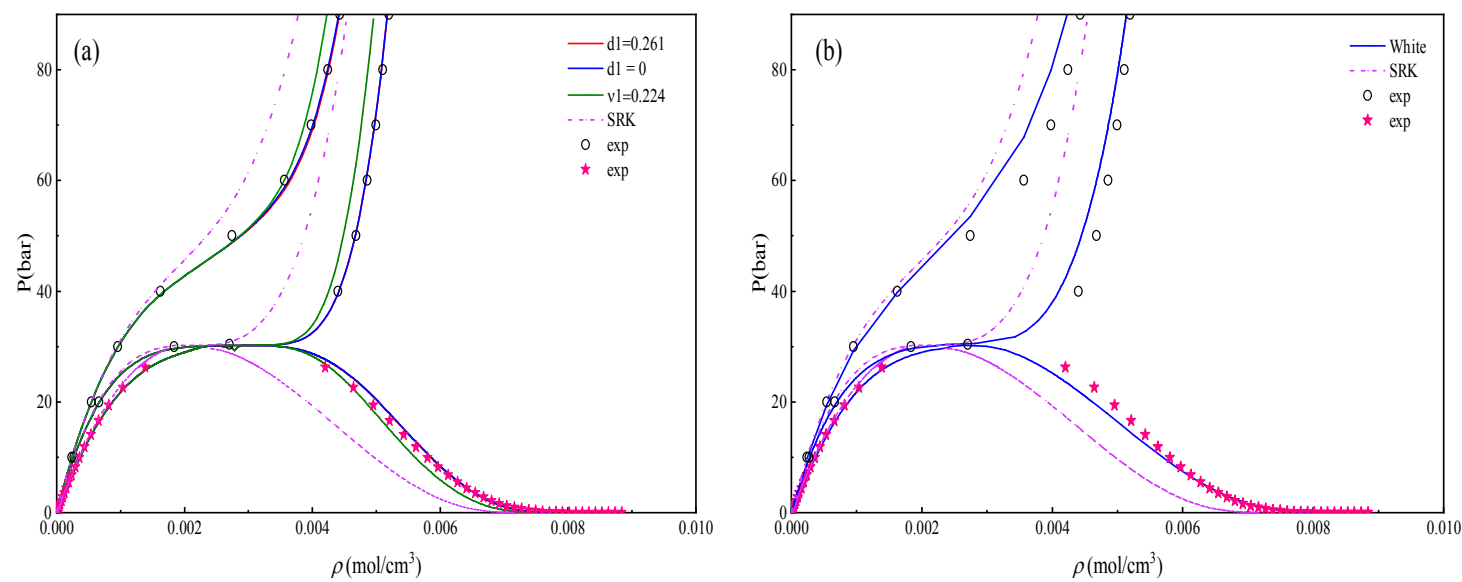

Fig. 4. (a) Influence of parameter $d_{1}$ and $v_{1}$ on the isotherms and saturated properties of n-hexane. Symbols are experimental data [39] and solid lines are crossover of SRK EoS based on Kiselev's procedure, (b) the isotherms and saturated properties of n-hexane based on White's procedure. 


\subsubsection{Method II}

In this method, we evaluated the influence of $v_{1}$ on the description of the VLE and PVT data. Therefore $d_{1}$ is considered equal to zero and the remaining parameters are re-optimized by fitting the experimental data according to the objective function, Eq. (32). The results of the parameter optimization are reported in Table 7 and the second columns of Table 6 . The value of $G i$ is restricted to less than 0.15 and the value of $v_{1}$ is smaller than $G i$. A simple correlation between the parameters and the molecular weight of the pure compounds is still no observed. However, there is not much difference between $G i$ and acentric factor values in comparison to Method I. Since $\Delta \eta_{c}$ is obtained after the adjustment of $v_{0 c}$, there is also a shift in the critical density; however, fixing $d_{1}$ equal to zero doesn't change the magnitude of $\Delta \eta_{c}$. Thus, $d_{1}$ has no significant role near the critical point and the results of critical parameters are reported in the second columns of Table 9, and for all cases, the accuracy of the critical pressure prediction is increased, but it does not have a significant effect on the critical temperature and volume.

Table 7. The values of adjusted parameters in the crossover SRK EoS using the second method of Kiselev's procedure for selected n-alkanes.

\begin{tabular}{|c|c|c|c|c|}
\hline Comp. & $\begin{array}{c}v_{0 c} \\
\left(\mathrm{~cm}^{3} / \mathrm{mol}\right)\end{array}$ & $\omega(-)$ & $G i(-)$ & $v_{1}(-)$ \\
\hline $\mathrm{C}_{1}$ & 125.655 & 0.016 & 0.050 & 0.026 \\
\hline $\mathrm{C}_{2}$ & 189.848 & 0.113 & 0.054 & 0.019 \\
\hline $\mathrm{C}_{3}$ & 251.127 & 0.169 & 0.098 & 0.017 \\
\hline $\mathrm{C}_{4}$ & 342.823 & 0.220 & 0.059 & 0.015 \\
\hline $\mathrm{C}_{5}$ & 431.848 & 0.273 & 0.061 & 0.014 \\
\hline $\mathrm{C}_{6}$ & 519.064 & 0.323 & 0.063 & 0.011 \\
\hline $\mathrm{C}_{7}$ & 611.466 & 0.375 & 0.066 & 0.010 \\
\hline $\mathrm{C}_{8}$ & 703.899 & 0.424 & 0.064 & 0.010 \\
\hline $\mathrm{C}_{9}$ & 725.823 & 0.469 & 0.120 & 0.007 \\
\hline $\mathrm{C}_{10}$ & 883.856 & 0.518 & 0.071 & 0.007 \\
\hline
\end{tabular}

\subsubsection{Method III}

Since the main effect on the vapor-liquid behavior is related to the classical parameters rather than crossover parameters, in this method the number of adjustment parameters are reduced to $G i, v_{0 c}$ and $\omega$ (the third columns of Table 8). So that, two additional constraints are considered: first, $d_{1}=0$; second, the parameter $v_{1}$ is given as linear function of the inverse $G i$, as suggested by Kiselev [17], and given by the expression:

$v_{1}=-4.9 \times 10^{-2}+\frac{0.005}{G i}$ 
The AAD\% for Method III are presented in the third columns of Tables 6 and 9. According to these tables, neglecting $d_{1}$ and constraining $v_{1}$ lead to an increase in the deviation of saturated properties and they have the main effect on the isothermal pressure-density data. Apart from the small quantitative differences, all methods can reasonably describe the saturated properties in the studied temperature and pressure ranges. In practice, there is no superiority for predicting the critical temperature and volume, but method III is superior to the others in representing the critical pressure (Fig. 5). It should be noted that the $\mathrm{AAD} \%$ of the critical temperature and volume in all cases of the three Kiselev's approaches are zero because they match the experimental values precisely.

Table 8. The values of adjusted parameters in the crossover SRK EoS using the third method of Kiselev's procedure for selected n-alkanes.

\begin{tabular}{|c|c|c|c|}
\hline Comp. & $\begin{array}{c}v_{0 c} \\
\left(\mathrm{~cm}^{3} / \mathrm{mol}\right)\end{array}$ & $\omega(-)$ & Gi $(-)$ \\
\hline $\mathrm{C}_{1}$ & 115.957 & 0.014 & 0.073 \\
\hline $\mathrm{C}_{2}$ & 171.700 & 0.106 & 0.080 \\
\hline $\mathrm{C}_{3}$ & 246.480 & 0.165 & 0.080 \\
\hline $\mathrm{C}_{4}$ & 316.230 & 0.214 & 0.083 \\
\hline $\mathrm{C}_{5}$ & 389.108 & 0.266 & 0.085 \\
\hline $\mathrm{C}_{6}$ & 481.190 & 0.318 & 0.086 \\
\hline $\mathrm{C}_{7}$ & 564.075 & 0.370 & 0.089 \\
\hline $\mathrm{C}_{8}$ & 664.250 & 0.424 & 0.087 \\
\hline $\mathrm{C}_{9}$ & 792.505 & 0.511 & 0.400 \\
\hline $\mathrm{C}_{10}$ & 837.439 & 0.515 & 0.091 \\
\hline
\end{tabular}

Table 9. AAD $\%$ of the calculated and experimental critical pressure of selected n-alkanes using the three methods for Kiselev's procedure.

\begin{tabular}{|c|c|c|c|}
\hline \multirow{2}{*}{ Comp. } & Method I & Method II & Method III \\
\hline & $\Delta P_{c}(\%)$ & $\Delta P_{c}(\%)$ & $\Delta P_{c}(\%)$ \\
\hline $\mathrm{C}_{1}$ & 0.00 & 0.07 & 0.00 \\
\hline $\mathrm{C}_{2}$ & 0.01 & 0.07 & 0.00 \\
\hline $\mathrm{C}_{3}$ & 0.07 & 0.01 & 0.00 \\
\hline $\mathrm{C}_{4}$ & 0.05 & 0.09 & 0.00 \\
\hline $\mathrm{C}_{5}$ & 0.13 & 0.12 & 0.00 \\
\hline $\mathrm{C}_{6}$ & 0.14 & 0.13 & 0.01 \\
\hline $\mathrm{C}_{7}$ & 0.57 & 0.12 & 0.00 \\
\hline $\mathrm{C}_{8}$ & 0.09 & 0.11 & 0.01 \\
\hline $\mathrm{C}_{9}$ & 0.09 & 0.00 & 0.07 \\
\hline $\mathrm{C}_{10}$ & 0.04 & 0.05 & 0.28 \\
\hline
\end{tabular}



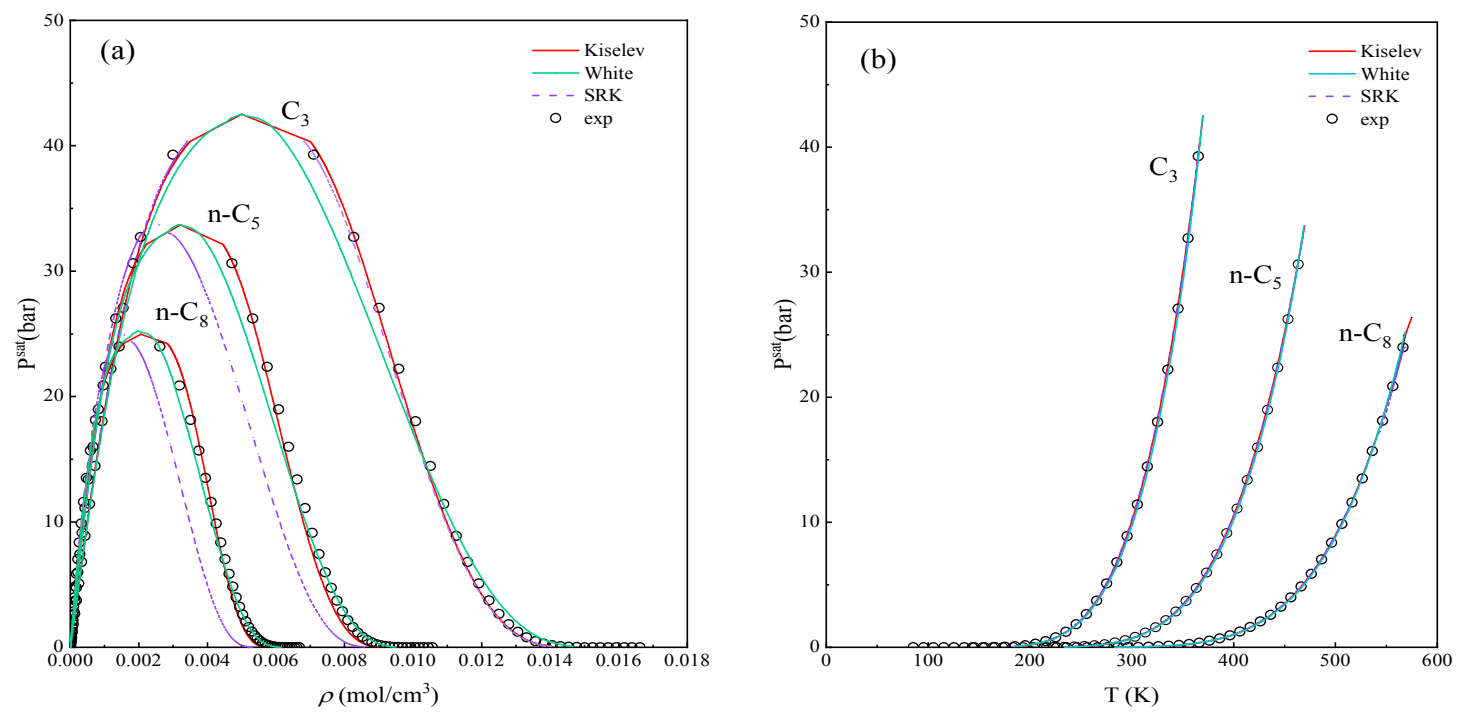

Fig. 5. (a) Phase diagrams and (b) saturated pressure for propane, n-pentane and n-octane according to Method III of Kiselev's procedure and Method I of White's procedure. Symbols are experimental data [39].

\section{Discussion}

Both crossover approaches, based on Kiselev's and White's procedures, yield significant improvement in terms of the accuracy regarding the reproduction of the saturated liquid phase density of the pure components in comparison to the classical SRK EOS. A similar behavior is observed for the isothermal pressure-density data, while no enhancements are seen for the saturated pressure (Table 10). The comparison of the critical deviations (Table 11) indicates that the third method of Kiselev's procedure is slightly inferior on critical volume of the systems. The analysis of the results given in the two tables shows that Kiselev's procedure is slightly superior to White's in the representation of the saturated pressure, especially near the critical point. The same is observed for the description of the critical isotherm close to the critical pressures and volumes. A reason for such behavior might be related to the larger amount of information used in the first method, even though, in the case of the third method the same number of adjustable parameters is used.

The comparison of the crossover parameters $G i$, and $v_{1}$ for the Methods II and III in Kiselev's procedure shows that the optimum values are similar and an analogous trend with respect to the molecular weight of the hydrocarbon (Fig. 3). The exception is n-nonane, which can be explained by the utilization of a subcritical isotherm in the objective function. Additionally, Table 10 shows that Method I of Kiselev's procedure yields better overall AAD\% for the isothermal pressure-density and 
saturated properties. This is because the additional parameters significantly increase the flexibility to fit the experimental data. However, due to the strong coupling between the parameters, it is harder to establish a function to relate the parameters with a pure component property, such as the molecular weight. Usually, the objective functions of complex models with several adjustable parameters possess different local minima, which might result in non-physical model parameters.

Table 10. AAD \% for saturated properties and isothermal pressure, Calculated Using the original SRK and CSRK EoS using White's and Kiselev's procedures.

\begin{tabular}{|c|c|c|c|c|c|}
\hline Procedure & Method & $\Delta P^{\text {sat }}(\%)$ & $\Delta \rho^{\text {liq }}(\%)$ & $\Delta \rho^{\text {vap }}(\%)$ & $\Delta P^{\text {isot }}(\%)$ \\
\hline \multirow{3}{*}{ White } & Method I & 2.13 & 2.30 & 1.75 & 7.24 \\
\cline { 2 - 6 } & Method II & 2.54 & 2.56 & 2.34 & 7.20 \\
\hline \multirow{3}{*}{ Kiselev } & Method I & 1.67 & 2.21 & 2.43 & 1.76 \\
\cline { 2 - 6 } & Method II & 1.72 & 2.44 & 3.27 & 6.30 \\
\cline { 2 - 6 } & Method III & 1.69 & 1.86 & 2.59 & 1.77 \\
\hline Original SRK & - & 1.64 & 13.07 & 1.64 & 53.0 \\
\hline
\end{tabular}

In the case of White's procedure, Fig. 1 and 2 indicate that a fewer number of parameters simplifies the optimization of the model, yielding classical critical parameters that follow a similar trend in comparison to the experimental properties. Besides, these parameters and the cutoff length can be regressed by a logarithmic function that can be used to predict the parameters of other molecules if experimental data is not available.

Since the original $S R K E O S$ was designed to match the critical temperature and pressure of the real fluid, the errors of these properties are zero for each component. Nevertheless, this model predicts a wrong critical volume with a large deviation. A substantial improvement in the representation of critical volume is obtained using the crossover procedures, despite the slight increase in the deviation of the critical temperature and pressure. The substantial improvement in the representation of critical volume is an indication of the non-mean-field character of the crossover procedures.

The models were also compared in terms of their capacity to predict the phase behavior of heavy hydrocarbons. This was done by extrapolating the parameters of the equations using correlations for the parameters with respect to the molecular weight of n-alkanes. Then, the predicted values were used in the models to calculate the saturated vapor pressures and liquid phase densities, as well as the critical point of the $\mathrm{C} 12, \mathrm{C} 14, \mathrm{C} 16$ and $\mathrm{C} 18$. Finally, the results were compared to the experimental data obtained from the DIPPR database [41]. 
Table 11. AAD\% for critical properties, Calculated Using the original SRK and CSRK EoS using White's and Kiselev's procedures.

\begin{tabular}{|c|c|c|c|c|}
\hline Procedure & Method & $\Delta T_{c}(\%)$ & $\Delta P_{c}(\%)$ & $\Delta \rho_{c}(\%)$ \\
\hline \multirow{3}{*}{ White } & Method I & 0.14 & 0.83 & 2.05 \\
\cline { 2 - 5 } & Method II & 0.20 & 0.28 & 0.57 \\
\hline \multirow{3}{*}{ Kiselev } & Method I & 0.00 & 0.12 & 0.00 \\
\cline { 2 - 5 } & Method II & 0.00 & 0.08 & 0.00 \\
\cline { 2 - 5 } & Method III & 0.00 & 0.04 & 0.00 \\
\hline SRK & - & 0.00 & 24.8 & 12.1 \\
\hline
\end{tabular}

As previously mentioned, a logarithmic function can be used to correlate with high precision the parameters of the Crossover SRK EoS using the second method of White's recursive procedure for $\mathrm{C} 2$ to $\mathrm{C} 10$. However, the extrapolation of the parameters to $\mathrm{n}$-dodecane and n-octadecane yields large deviations regarding the prediction of the phase equilibrium properties of the two components. Therefore, a different approach was utilized, similar to the one described in reference [40].
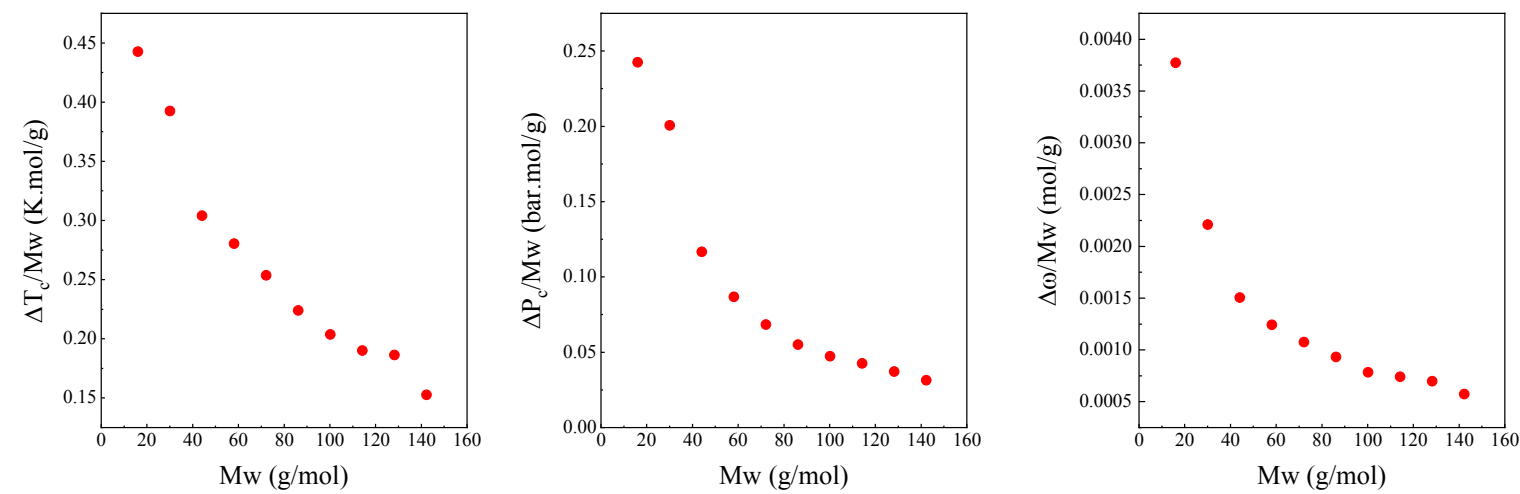

Fig. 6. The difference between the classical and virtual parameters for the crossover SRK using White's recursive procedure as a function of the molecular weight of selected n-alkanes.

In this procedure, we plotted the difference between the experimental and classical parameters (Fig. 6) for methane to n-decane. Then, a power law was used to regress the data, yielding the following expressions:

$$
\begin{aligned}
& \Delta T_{0 c}=\left|T_{0 c}-T_{c}\right|=1.848(M w)^{0.522} \\
& \Delta P_{0 c}=\left|P_{0 c}-P_{c}\right|=4.717(M w)^{0.006} \\
& \Delta \omega_{0}=\left|\omega_{0}-\omega\right|=0.0371(M w)^{0.169}
\end{aligned}
$$


where $\Delta T_{0 c}, \Delta P_{0 c}$ and $\Delta \omega_{0}$ are the difference between the critical temperatures, pressures, and acentric factors, respectively, from $S R K$ and crossover $S R K$. The classical parameters of the crossover model were predicted for $\mathrm{C} 12$ to $\mathrm{C} 18$ using the following equations.

$T_{0 c}=T_{c}+\Delta T_{0 c}$

$P_{0 c}=P_{c}+\Delta P_{0 c}$

$\omega_{0}=\omega+\Delta \omega_{0}$

Thereafter, the cutoff lengths were predicted using its correlation with the molecular weight

$L=1.598 \ln M w-0.7287$

and $\phi$ was considered equal to 1.5 . This procedure led to much better results.

Fig. 7 shows the saturated vapor pressures and liquid phase densities curves predicted by the crossover $S R K E o S$, and the experimental values from the DIPPR database [41]. It is possible to observe that the non-mean-field thermodynamic model accurately describes the data, although the deviations with respect to the critical point increase with the molecular weight. Table 12 shows that the deviations between the model and experiments for the saturated and critical properties. In the first case, the overall AAD for $P^{v a p}$ is around $3 \%$, while for $v^{l i q}$ is below this value. The deviations for $T_{c}$ and $\rho_{c}$ are below $0.5 \%$ and $1.5 \%$, respectively. On the other hand, a larger error is observed for $P_{c}$, e.g. $8.74 \%$ for $\mathrm{C} 18$. Nevertheless, the uncertainty of the experimental data is larger than the calculated deviations, e.g. $25 \%$ for $\mathrm{C} 18$. In summary, the results indicate that the parameters of the model can be extrapolated to other substances in the same family, yielding accurate representations.

The crossover model based on Kiselev's procedure and with Method III was also applied in the prediction of the properties of the heavy hydrocarbons mentioned previously. The parameters were initially estimated by regressing the data plotted in Fig. 3, excluding n-nonane, yielding the following expressions:

$$
\begin{aligned}
& v_{0 c}=5.7752 M w-4.9614 \\
& \Delta \omega_{0}=\left|\omega_{0}-\omega\right|=0.0002(M w)^{1.0765} \\
& G i=0.0571 M w^{0.0928}
\end{aligned}
$$



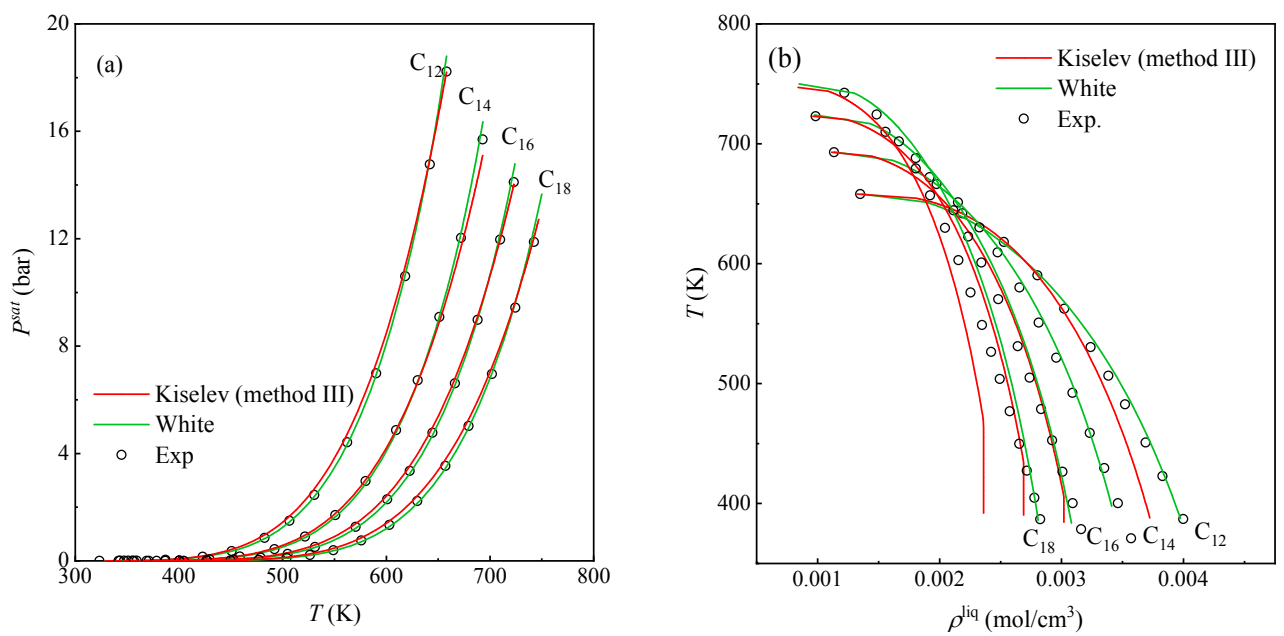

Fig. 7. (a) Saturated pressure and (b) Saturated liquid density for selected heavier n-alkanes using the extrapolation of the correlations between the crossover parameters and molecular weight. Symbols are experimental data taken from the DIPPR database [41].

The previous equations were applied to predict the input parameters of the model. Thereafter, it was used to represent the saturated and critical properties of $\mathrm{C} 12$ to $\mathrm{C} 18$. Fig. 7 shows that the crossover model precisely matches the critical point of the fluids, although large deviations are observed for the saturated properties. The same is seen in Table 12, which shows an overall AAD\% of $10.2 \%$ for $P^{v a p}$, and $8 \%$ for $\rho^{l i q}$. Similar to the other non-mean-field equation, a power law was used to extrapolate the values for the acentric factor, which improved the representations of the saturated properties, but still large deviations were found.

Table 12. AAD\% between the calculated and experimental saturated and critical properties of heavier n-alkanes using the extrapolation of the correlations between the crossover parameters and molecular weight.

\begin{tabular}{|c|c|c|c|c|c|c|c|c|c|c|}
\hline \multirow[b]{2}{*}{ Comp. } & \multicolumn{5}{|c|}{ Kiselev's procedure } & \multicolumn{5}{|c|}{ White's procedure } \\
\hline & $\begin{array}{l}\Delta P^{\text {sat }} \\
(\%)\end{array}$ & $\begin{array}{l}\Delta \rho^{\text {liq }} \\
(\%)\end{array}$ & $\begin{array}{l}\Delta T_{c} \\
(\%) \\
\end{array}$ & $\begin{array}{l}\Delta P_{c} \\
(\%)\end{array}$ & $\begin{array}{l}\Delta \rho_{c} \\
(\%)\end{array}$ & $\begin{array}{l}\begin{array}{l}\Delta P^{\text {sat }} \\
(\%)\end{array} \\
\end{array}$ & $\begin{array}{l}\Delta \rho^{\text {liq }} \\
(\%)\end{array}$ & $\begin{array}{l}\Delta T_{c} \\
(\%)\end{array}$ & $\begin{array}{l}\Delta P_{c} \\
(\%) \\
\end{array}$ & $\begin{array}{l}\Delta \rho_{c} \\
(\%)\end{array}$ \\
\hline $\mathrm{C} 12$ & 10.49 & 4.55 & 0.00 & 0.00 & 0.00 & 2.14 & 1.36 & 0.00 & 3.15 & 1.13 \\
\hline $\mathrm{C} 14$ & 6.44 & 10.20 & 0.00 & 0.18 & 0.00 & 2.91 & 1.4 & 0.02 & 4.20 & 0.20 \\
\hline $\mathrm{C} 16$ & 10.01 & 8.38 & 0.00 & 0.10 & 0.00 & 2.39 & 2.03 & 0.15 & 4.80 & 0.98 \\
\hline $\mathrm{C} 18$ & 13.97 & 8.83 & 0.00 & 0.16 & 0.00 & 4.78 & 4.08 & 0.39 & 8.74 & 2.57 \\
\hline Average & 10.23 & 7.99 & 0.00 & 0.11 & 0.00 & 3.06 & 2.22 & 0.14 & 5.23 & 1.22 \\
\hline
\end{tabular}

The comparison of the two crossover models for predicting the properties of heavy hydrocarbons denotes that the extrapolation of the input parameters from lighter n-alkanes is possible. However, a careful analysis of the correlations with the molecular weight is necessary, in order to achieve accurate 
results. On the other hand, the correlation used with the crossover SRK EOS using the third method of Kiselev's approach did not yield precise descriptions of the experimental saturated data, which indicates that further investigations are necessary, especially for the methods with more adjustable parameters to extrapolate the parameters for the substances in the same homologous series.

Finally, a crucial test of the studied procedures to represent the non-analytical behavior of fluids close to the critical point is the calculation of the critical exponents. Mean-field methods are known to be unable to predict the correct values of the universal critical exponents. This is due to the fact that they do not take into account the long-range interactions that arise close to the critical point in the range $10^{-4}<|\Delta T|<0.02$. In this work, we have calculated the $\beta$ exponent using the following expression:

$$
\ln \left[\left(\rho_{l}-\rho_{v}\right) / \rho_{c}\right]=\beta \ln \left[\left(T-T_{c}\right) / T_{c}\right]+C
$$

The results are shown in Fig. 8. Both procedures give acceptable values for $\beta$ that are close to the universal one, 0.326, while the classical SRK results in a value close to 0.5. It is also seen that Kiselev's procedure has a wider scattering and all above 0.326 , revealing that the approach corrects the asymptotic scaling in a significant manner but not completely (mean-field value is 0.5 and some provided values are 0.4 ). The results from White's procedure are on average slightly better and the Method I of White's approach reveals that the critical exponent decreases with the increase of the chain length of the n-alkane. This produces an excessive flattening of the critical region, which is a phenomenon observed for long alkanes in other contributions who have used this approach. Therefore, it is concluded that both models account for the asymptotic and singular critical behavior of real fluids. 

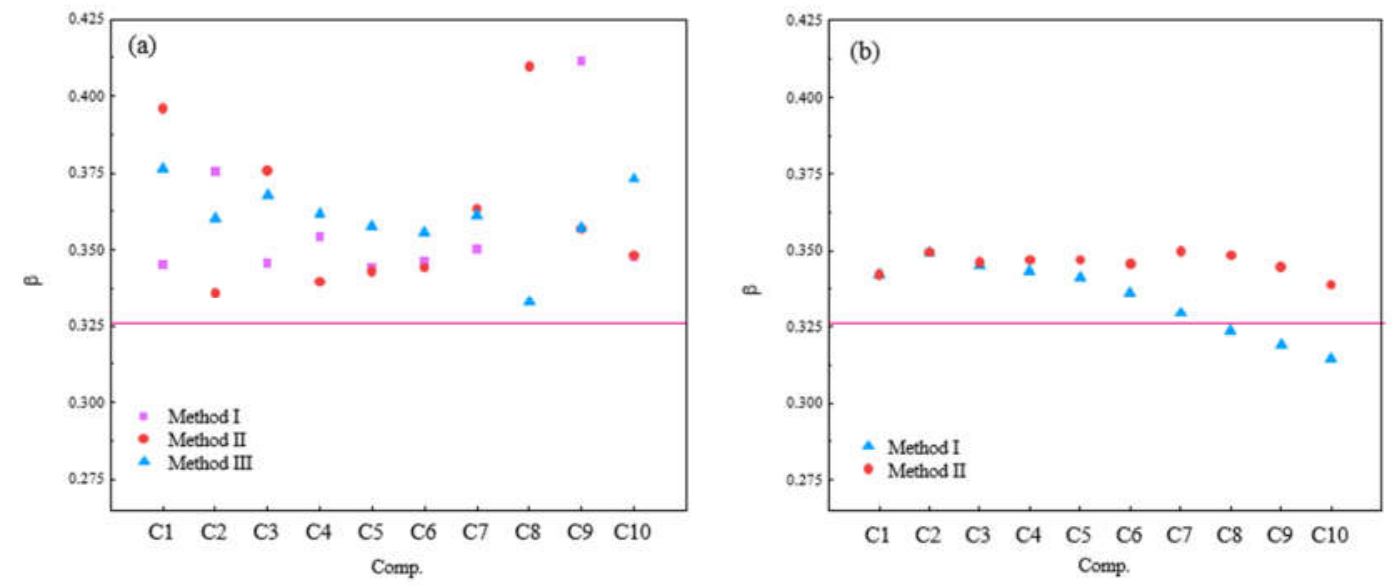

Fig. 8. Evaluation of $\beta$ as one of critical exponent for pure fluids, (left side) using Methods I, II and III based on Kiselev's procedure, (right side) using Methods I and II based on White's procedure.

\section{Conclusion}

In this work, the comparison of two procedures is presented to introduce the long wavelength fluctuations close to the critical point into the classical $S R K E O S$, namely Kiselev's and White's procedure. Kiselev's procedure has a closed analytical expression for Helmholtz free energy and pressure whereas White's procedure needs to be solved numerically and requires an additional spline function for a representation of the thermodynamic surface of real fluids. Due to these features, Kiselev's procedure is approximately two times faster than White's.

The two procedures were applied to the representation of the $P V T$ data along two isotherms, one critical and one super-critical, and of saturated and critical properties for n-alkanes (from methane to n-decane). The results obtained for the description of PVT data have shown that the two crossover procedures are capable of precisely representing the isothermal pressure curves along critical and supercritical temperatures, since the deviations from experimental data obtained with these nonclassical equations are much smaller than those from the classical SRKEOS. In the case of the saturated properties, including saturated vapor pressures, the presented results showed that two procedures could describe the experimental data as well as classical $S R K E O S$. For critical properties, two procedures could correctly represent critical points; nevertheless, Kiselev's procedure perfectly matches these properties particularly using Method III by restricting two crossover parameters, i.e. $d_{1}$ and $v_{1}$, while White's procedure yields small deviations. Finally, the comparison of the model to predict the saturated and critical properties of heavy n-alkanes (from methane to n-decane) indicates 
that the estimation of the input parameter can be done from the correlations regarding the lighter hydrocarbons, as long as a careful analysis of the results is applied. Yet, precise results for the saturated properties were only obtained from the crossover model with the recursive procedure, indicating that further evaluations for the Kiselev's procedure are required to develop a function to extrapolate the input parameters within the same homologous series.

\section{- Associated Content: Supplementary data}

- Expressions of classical Helmholtz free energy for Kiselev's procedure

- Expressions of Crossover Helmholtz free energy for SRK EoS based on the Kiselev's procedure

- The analytic expression for pressure based on the Kiselev's procedure

\section{Acknowledgement}

The authors gratefully acknowledge the financial support from Center for Energy Resources Engineering (CERE) at Technical University of Denmark (DTU), the Science Without Borders Program of the Brazilian Ministry of Education for paying the scholarship of Andre P.C.M. Vinhal and the Research Committee of University of Tehran. 


\section{Notations:}

\section{List of Symbols}

A

$\bar{A} \quad$ dimensionless Helmholtz free energy

a temperature dependent of attraction parameter

$a_{0} \quad$ system dependent parameter

b system dependent parameter

$b^{2} \quad$ universal linear model parameter

$d_{1} \quad$ rectilinear diameter amplitude

Gi Ginzburg number

$k$

$p^{2}$

$P$

$q$

$R$

$T$

$\Delta T$

$\bar{T}$

$v$

$\bar{v}$

$v_{1}$

$\Delta v$

Y

$F_{S}$

$f^{s}$

$f^{n}$

a component specific parameter

universal sine model parameter

pressure (bar)

argument of crossover function

gas constant (bar. $\mathrm{cm}^{3} / \mathrm{mol}$. K)

temperature $(\mathrm{K})$ critical temperature

rescaled value of temperature

molar volume

rescaled value of volume

system dependent coefficient

classical order parameter

crossover function

short wavelength fluctuations

dimensionless deviation of the temperature from the classical

the Helmholtz free energy density for a homogeneous system

the free energy density incorporating the density fluctuations

on all wavelength scales

\section{Greek symbols}

$\alpha$

universal critical constant 


$\begin{array}{ll}\beta & \text { universal critical constant } \\ \gamma & \text { universal critical constant } \\ \Delta_{1} & \text { universal critical constant } \\ \eta & \text { reduced volume difference } \\ \bar{\eta} & \text { rescaled order parameter } \\ \tau & \text { reduced temperature difference } \\ \bar{\tau} & \text { rescaled reduced temperature difference } \\ \omega & \text { acentric factor } \\ \Omega^{s} & \text { the density fluctuations for the short range attraction } \\ \Omega^{l} & \text { the density fluctuations for the long range attraction } \\ \phi & \text { an adjustable parameter } \\ w & \text { the range of the attractive potential }\end{array}$

\section{Superscripts}

$\begin{array}{ll}\text { calc } & \text { calculated } \\ \exp & \text { experimental } \\ \text { ideal } & \text { ideal } \\ \text { isot } & \text { isothermal } \\ \text { res } & \text { residual } \\ \text { sat } & \text { saturated } \\ \text { s } & \text { short range attraction } \\ 1 & \text { long range attraction }\end{array}$

\section{Subscripts}

0c

classical

c

critical 


\section{References}

[1] J. V Sengers, J.M.H.L. Sengers, Thermodynamic behavior of fluids near the critical point, Annu. Rev. Phys. Chem. 37 (1986) 189-222.

[2] S.B. Kiselev, J. V Sengers, An improved parametric crossover model for the thermodynamic properties of fluids in the critical region, Int. J. Thermophys. 14 (1993) 1-32.

[3] S. Horstman, Theoretische und Experimentelle Untersuchungen zum Hochdruckphasengleichgewichtsverhalten Fluider Stoffgemische für die Er- weiterung der PSRK-Gruppenbeitragszustandsgleichung, 2000.

[4] M.A. Anisimov, J. V. Sengers, Critical and Crossover Phenomena in Fluids and Fluid Mixtures, in: Kiran, E., P.G. Debenedetti, C.J. Peters (Eds.), Supercritical Fluids Fundam. Appl., Springer Netherlands, 2000: pp. 89-121.

[5] G. Pérez-Sánchez, P. Losada-Pérez, C.A. Cerdeiriña, J. V Sengers, M.A. Anisimov, Asymmetric criticality in weakly compressible liquid mixtures, J. Chem. Phys. 132 (2010) 154502. doi:10.1063/1.3378626.

[6] A. Kostrowicka Wyczalkowska, M.A. Anisimov, J. V Sengers, Global crossover equation of state of a van der Waals fluid, Fluid Phase Equilib. 158-160 (1999) 523-535. doi:https://doi.org/10.1016/S0378-3812(99)00070-9.

[7] S.B. Kiselev, D.G. Friend, Cubic crossover equation of state for mixtures1, Fluid Phase Equilib. 162 (1999) 51-82. doi:https://doi.org/10.1016/S0378-3812(99)00182-X.

[8] S.B. Kiselev, Cubic crossover equation of state1, Fluid Phase Equilib. 147 (1998) 7-23. doi:https://doi.org/10.1016/S0378-3812(98)00222-2.

[9] S.B. Kiselev, J.F. Ely, Simplified crossover SAFT equation of state for pure fluids and fluid mixtures, Fluid Phase Equilib. 174 (2000) 93-113. doi:https://doi.org/10.1016/S03783812(00)00420-9.

[10] L.A. Forero G, J.A. Velásquez J, A generalized cubic equation of state for non-polar and polar substances, Fluid Phase Equilib. 418 (2016) 74-87. doi:https://doi.org/10.1016/j.fluid.2015.09.045.

[11] G.M. Kontogeorgis, I.G. Economou, Equations of state: From the ideas of van der Waals to association theories, J. Supercrit. Fluids. 55 (2010) 421-437. doi:https://doi.org/10.1016/j.supflu.2010.10.023.

[12] Z.Y. Chen, P.C. Albright, J. V Sengers, Crossover from singular critical to regular classical thermodynamic behavior of fluids, Phys. Rev. A. 41 (1990) 3161-3177. doi:10.1103/PhysRevA.41.3161.

[13] A.K. Wyczalkowska, J. V Sengers, M.A. Anisimov, Critical fluctuations and the equation of state of Van der Waals, Phys. A Stat. Mech. Its Appl. 334 (2004) 482-512. doi:https://doi.org/10.1016/j.physa.2003.11.021.

[14] S.B. Kiselev, I.G. Kostyukova, A.A. Povodyrev, Universal crossover behavior of fluids and fluid mixtures in the critical region, Int. J. Thermophys. 12 (1991) 877-895.

[15] S.B. Kiselev, J.F. Ely, Crossover SAFT equation of state: application for normal alkanes, 
Ind. Eng. Chem. Res. 38 (1999) 4993-5004.

[16] S.B. Kiselev, J.F. Ely, I.M. Abdulagatov, J.W. Magee, Crossover SAFT equation of state and thermodynamic properties of propan-1-ol, Int. J. Thermophys. 21 (2000) 1373-1405.

[17] S.B. Kiselev, J.F. Ely, H. Adidharma, M. Radosz, A crossover equation of state for associating fluids, Fluid Phase Equilib. 183 (2001) 53-64.

[18] C. McCabe, S.B. Kiselev, A crossover SAFT-VR equation of state for pure fluids: preliminary results for light hydrocarbons, Fluid Phase Equilib. 219 (2004) 3-9.

[19] J.A. White, S. Zhang, Renormalization group theory for fluids, J. Chem. Phys. 99 (1993) 2012-2019. doi:10.1063/1.465263.

[20] J.A. White, Contribution of fluctuations to thermal properties of fluids with attractive forces of limited range: theory compared with $\mathrm{P} Q \mathrm{~T}$ and $\mathrm{Cv}$ data for argon, Fluid Phase Equilib. 75 (1992) 53-64. doi:https://doi.org/10.1016/0378-3812(92)87006-9.

[21] G.M. Wilson, Vapor-liquid equilibrium. XI. A new expression for the excess free energy of mixing, J. Am. Chem. Soc. 86 (1964) 127-130.

[22] J. Jiang, J.M. Prausnitz, Equation of state for thermodynamic properties of chain fluids nearto and far-from the vapor-liquid critical region, J. Chem. Phys. 111 (1999) 5964-5974. doi:10.1063/1.479892.

[23] J. Jiang, J.M. Prausnitz, Critical temperatures and pressures for hydrocarbon mixtures from an equation of state with renormalization-group theory corrections, Fluid Phase Equilib. 169 (2000) 127-147. doi:https://doi.org/10.1016/S0378-3812(00)00299-5.

[24] F. Llovell, L.F. Vega, Prediction of thermodynamic derivative properties of pure fluids through the soft-SAFT equation of state, J. Phys. Chem. B. 110 (2006) 11427-11437.

[25] L.W. Salvino, J.A. White, Calculation of density fluctuation contributions to thermodynamic properties of simple fluids, J. Chem. Phys. 96 (1992) 4559-4568.

[26] M. Dicko, C. Coquelet, Application of a new crossover treatment to a generalized cubic equation of state, Fluid Phase Equilib. 302 (2011) 241-248. doi:https://doi.org/10.1016/j.fluid.2010.10.026.

[27] J. Janeček, P. Paricaud, M. Dicko, C. Coquelet, A generalized Kiselev crossover approach applied to Soave-Redlich-Kwong equation of state, Fluid Phase Equilib. 401 (2015) 16-26. doi:https://doi.org/10.1016/j.fluid.2015.04.024.

[28] A. Anderko, Cubic and generalized van der Waals equations, in: J. Sengers, R. Kayser, C. Peters, H. White (Eds.), Equations State Fluids Fluid Mix., 2000: pp. 75-126.

[29] G. Soave, Equilibrium constants from a modified Redlich-Kwong equation of state, Chem. Eng. Sci. 27 (1972) 1197-1203.

[30] F. Llovell, J.C. Pàmies, L.F. Vega, Thermodynamic properties of Lennard-Jones chain molecules: Renormalization-group corrections to a modified statistical associating fluid theory, J. Chem. Phys. 121 (2004) 10715-10724. doi:10.1063/1.1809112.

[31] L. Lue, J.M. Prausnitz, Renormalization-group corrections to an approximate free-energy model for simple fluids near to and far from the critical region, J. Chem. Phys. 108 (1998) 
$5529-5536$.

[32] J. Cai, J.M. Prausnitz, Thermodynamics for fluid mixtures near to and far from the vaporliquid critical point, Fluid Phase Equilib. 219 (2004) 205-217. doi:10.1016/j.fluid.2004.01.033

[33] J. Jiang, J.M. Prausnitz, Critical temperatures and pressures for hydrocarbon mixtures from an equation of state with renormalization-group theory corrections, Fluid Phase Equilib. 169 (2000) 127-147. doi:https://doi.org/10.1016/S0378-3812(00)00299-5.

[34] F. Llovell, L.F. Vega, Global fluid phase equilibria and critical phenomena of selected mixtures using the crossover soft-SAFT equation, J. Phys. Chem. B. 110 (2006) 1350-1362. doi:10.1021/jp0551465.

[35] A.P.C.M. Vinhal, W. Yan, G.M. Kontogeorgis, Application of a Crossover Equation of State to Describe Phase Equilibrium and Critical Properties of n-Alkanes and Methane/n-Alkane Mixtures, J. Chem. Eng. Data. 63 (2018) 13. doi:10.1021/acs.jced.7b00779.

[36] K.G. Wilson, Renormalization Group and Critical Phenomena. I. Renormalization Group and the Kadanoff Scaling Picture, Phys. Rev. B. 4 (1971) 3174-3183. doi:10.1103/PhysRevB.4.3174.

[37] K.G. Wilson, M.E. Fisher, Critical Exponents in 3.99 Dimensions, Phys. Rev. Lett. 28 (1972) 240-243. doi:10.1103/PhysRevLett.28.240.

[38] M.A. Anisimov, J. Wang, Nature of Asymmetry in Fluid Criticality, Phys. Rev. Lett. 97 (2006) 025703. doi:10.1103/PhysRevLett.97.025703.

[39] M. Lemmon, E. , Huber, M., McLinden, NIST, Standard Reference Data Program, Gaithersburg. (2013).

[40] F. Llovell, L.F. Vega, D. Seiltgens, A. Mejía, H. Segura, An accurate direct technique for parametrizing cubic equations of state. Part III. Application of a crossover treatment, Fluid Phase Equilib. 264 (2008) 201-210. doi:10.1016/j.fluid.2007.11.006

[41] R.L. Rowley; W.V. Wilding, J.L. Oscarson; N.A. Zundel; T.L. Marshall; T.E. Daubert, R.P. Danner, DIPPR Data Compilation of Pure Compound Properties; Design Institute for Physical Properties, AIChE: New York, 2002.

[42] A. Bymaster, C. Emborsky, A. Dominik, W.G. Chapman, Renormalization-Group Corrections to a Perturbed-Chain Statistical Associating Fluid Theory for Pure Fluids Near to and Far from the Critical Region. Ind. Eng. Chem. Res. 2008, 47, 6264-6274. doi: $10.1021 /$ ie 8001167

[43] X. Tang, J. Gross, Renormalization-Group Corrections to the Perturbed-Chain Statistical Associating Fluid Theory for Binary Mixtures. Ind. Eng. Chem. Res. 2010, 49, 9436-9444. doi: $10.1021 /$ ie $100890 \mathrm{~d}$ 